\title{
Cuckoo's eggs in neutron stars: can LIGO hear chirps from the dark sector?
}

\author{
Joachim Kopp, ${ }^{a, b}$ Ranjan Laha, ${ }^{a}$ Toby Opferkuch $^{a}$ and William Shepherd ${ }^{a}$ \\ ${ }^{a}$ PRISMA Cluster of Excellence, Johannes Gutenberg-Universität Mainz, \\ Staudingerweg 9, Mainz, 55128, Germany \\ ${ }^{b}$ Theoretical Physics Department, CERN, \\ 1211 Geneva, Switzerland \\ E-mail: jkopp@uni-mainz.de, ranjalah@uni-mainz.de, \\ opferkuch@uni-mainz.de, shepherd@uni-mainz.de
}

ABSTRACT: We explore in detail the possibility that gravitational wave signals from binary inspirals are affected by a new force that couples only to dark matter particles. We discuss the impact of both the new force acting between the binary partners as well as radiation of the force carrier. We identify numerous constraints on any such scenario, ultimately concluding that observable effects on the dynamics of binary inspirals due to such a force are not possible if the dark matter is accrued during ordinary stellar evolution. Constraints arise from the requirement that the astronomical body be able to collect and bind at small enough radius an adequate number of dark matter particles, from the requirement that the particles thus collected remain bound to neutron stars in the presence of another neutron star, and from the requirement that the theory allows old neutron stars to exist and retain their charge. Thus, we show that any deviation from the predictions of general relativity observed in binary inspirals must be due either to the material properties of the inspiraling objects themselves, such as a tidal deformability, to a true fifth force coupled to baryons, or to a non-standard production mechanism for the dark matter cores of neutron stars. Viable scenarios of the latter type include production of dark matter in exotic neutron decays, or the formation of compact dark matter objects in the early Universe that later seed star formation or are captured by stars.

Keywords: Beyond Standard Model, Cosmology of Theories beyond the SM

ARXIV EPRINT: 1807.02527 


\section{Contents}

1 Introduction 1

2 Inspirals and fifth-forces $\quad 2$

2.1 Effects of a new Yukawa force 3

$\begin{array}{ll}2.2 & \text { Ultra-light boson radiation }\end{array}$

$\begin{array}{lll}3 & \text { Constraints on repulsive forces } & 8\end{array}$

3.1 Binding potential for DM particles 9

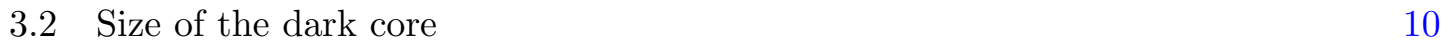

$\begin{array}{lll}3.3 & \text { Constraints on dark core production via particle accretion } & 12\end{array}$

$\begin{array}{ll}\text { 3.4 Constraints on dark core production in supernovae } & 13\end{array}$

$\begin{array}{ll}\text { 3.5 Constraints on dark core production via neutron decay } & 14\end{array}$

4 Constraints on attractive forces $\quad 16$

$\begin{array}{ll}4.1 \text { Black hole formation } & 16\end{array}$

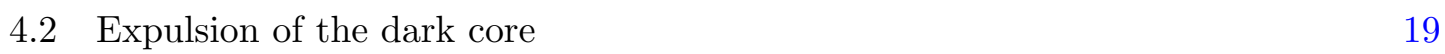

$\begin{array}{ll}4.3 \text { Constraints on dark core production mechanisms } & 20\end{array}$

5 Conclusions $\quad 21$

$\begin{array}{ll}\text { A Capture rate calculation } & 23\end{array}$

\section{Introduction}

The dawn of gravitational wave (GW) astronomy, commencing with the measurement of GWs produced by coalescing binary black holes (BHs), promises an entirely new era of observational astrophysics [1-3]. Recently, coalescing neutron star binaries have also been observed for the first time [4]. Such events are treasure troves of new data for astrophysics and astroparticle physics. They not only feature an associated electromagnetic signal [5-7], but also produce gravitational waveforms that are potentially observable over time periods of order 20 minutes and at length scales which are different from observations of binary pulsars [8].

The ability to track the neutron star binary inspiral over an extended period of time provides increased sensitivity to modifications of the post-Newtonian phase of the inspiral compared to inspirals of $\mathcal{O}\left(30 M_{\odot}\right)$ black holes. These deviations can be broadly categorized as stemming either from (i) a modification of gravity [9-18], (ii) a "fifth force" mediated by particles such as axions [19] or ultra-light dark gauge bosons [20], or from (iii) a new longrange force coupled to dark matter (DM) particles accreted inside neutron stars [21-23]. 
Stringent constraints exist on a new fifth force coupled to baryons, see for instance refs. [2428 ] and references therein, which have motivated a particular focus on the possibility of a new long-range force, mediated by ultra-light bosons, that couples only to dark matter or other cosmologically stable hidden sector particles residing inside neutron stars. Such scenarios have been studied extensively using probes other than gravitational waves, in particular the properties (and the mere existence) of old neutron stars and white dwarfs [2954] as well as the dynamics of binary pulsar PSR 1913+16 (Hulse-Taylor binary) [55, 56]. We note that dark sector particles experiencing a strong long-range force can at most contribute a small fraction to the total DM density of the Universe due to tight constraints on DM self-interactions [57-59].

Complementing these results, we will show that DM accumulation in neutron stars throughout the course of ordinary stellar evolution can never be efficient enough for the new force to lead to observable deviations in gravitational wave signals from binary inspirals. Detectable, per cent-level deviations require $\gtrsim 10 \%$ of the neutron star mass to be made up of dark sector particles. Exploring the constraints on the amount of such particles that a neutron star can contain, we will conclude that only exotic neutron decays or the formation of dark stars can result in such large abundances.

We begin in section 2 by reviewing fifth-force effects in neutron star binary inspirals, focusing on the required region of parameter space to observe deviations from general relativity in LIGO/VIRGO data. Of course, our results are easily generalized to other gravitational wave detectors such as ALIA, BBO, DECIGO, the Einstein Telescope, Geo600, KAGRA, LISA, TAMA, etc. [60, 61]. We then derive constraints on these forces, distinguishing between repulsive forces (section 3) and attractive ones (section 4). We conclude in section 5. Note that we use natural units throughout the text.

\section{Inspirals and fifth-forces}

Before turning to the constraints on the interactions of any exotic hidden sector particle, we first illustrate the necessary conditions for an observable new physics signal at LIGO/VIRGO. In doing so, we closely follow refs. [11, 20]. To remain as model-independent as possible, we parameterize the effect of the new dark force using a generic Yukawa potential. We furthermore assume that the new force couples only to dark sector particles. In order for it to act over sufficiently long distances of order $100 \mathrm{~km}$, the mediator must be ultra-light $\left(\lesssim 10^{-12} \mathrm{eV}\right)$, and the dark sector particles must neither significantly screen the new force nor efficiently self-annihilate. The most straightforward way of realizing the second constraint is asymmetric DM $[62,63]$.

Let us consider a binary neutron star system, and let us assume that each of the binary partners contains a population of dark sector particles. These populations will affect the inspiral dynamics in two distinct ways: first, the exotic force acting between them will affect the time evolution of the distance between the neutron stars, their orbital frequency, and the time of the merger. Second, radiation of the new light force carriers provides an extra energy loss mechanism. 


\subsection{Effects of a new Yukawa force}

Even in the presence of a new dark sector Yukawa force, the system's gravitational wave emission will follow the predictions of general relativity as long as the distance between the binary partners is significantly larger than the range of that force. However, once their distance drops below that range and the exponential suppression of the Yukawa potential is lifted, observable deviations may occur. The assumption of a Yukawa force is necessary, as the potential effects of a new infinite range force are degenerate with a shift of the neutron star masses. The new force, which can be either attractive or repulsive, results in a modification of the chirp mass $\mathcal{M}_{c} \equiv \mu^{3 / 5}\left(M_{1}+M_{2}\right)^{2 / 5}$, where $\mu=M_{1} M_{2} /\left(M_{1}+M_{2}\right)$ is the reduced mass of the inspiraling objects with individual masses $M_{1}$ and $M_{2}$. Assuming point-like neutron stars, we can write the magnitude of the force between the two bodies as

$$
|\mathbf{F}|=\frac{G_{N} M_{1} M_{2}}{\Delta^{2}}\left[1+\widetilde{\alpha}^{\prime} e^{-m_{\text {med }} \Delta}\left(1+m_{\text {med }} \Delta\right)\right],
$$

where $\Delta$ is their spatial separation and $m_{\text {med }}$ is the mass of the particle sourcing the Yukawa potential. Here $\widetilde{\alpha}^{\prime}$ parameterizes the size of the new force relative to gravity in the regime $m_{\text {med }} \Delta \ll 1$ :

$$
\widetilde{\alpha}^{\prime} \equiv \pm \frac{\alpha^{\prime} Q_{1} Q_{2}}{G_{N} M_{1} M_{2}}
$$

The sign in this expression determines whether the force is attractive (positive sign) or repulsive (negative sign). $\alpha^{\prime}$ is the analog of the fine structure constant for the new force, and $Q_{i}=q_{\chi} N_{\chi i}$ are the total charges of the inspiraling objects under the new force, which depend on the charge $q_{\chi}$ of a single DM particle and the numbers $N_{\chi i}$ of captured DM particles. Given that the orbits of the binary system will have circularized by the time the gravitational wave signal becomes observable [64], the orbital frequency of the system is given simply by a modified Kepler's law, ${ }^{1}$

$$
\omega^{2}=\frac{G_{N}\left(M_{1}+M_{2}\right)}{\Delta^{3}}\left[1+\widetilde{\alpha}^{\prime} e^{-m_{\text {med }} \Delta}\left(1+m_{\text {med }} \Delta\right)\right] .
$$

The frequency of the gravitational wave signal is $f_{\mathrm{GW}}=\omega / \pi$; therefore if LIGO sensitivity begins at $\mathcal{O}(10 \mathrm{~Hz})$ [4], eq. (2.3) tells us that a binary system consisting of two $1.25 M_{\odot}$ neutron stars enters the sensitivity band at a spatial separation of $\mathcal{O}(700 \mathrm{~km})$ (assuming that gravity is the dominant force at these length scales, i.e. $\left.\left|\widetilde{\alpha}^{\prime}\right| \ll 1\right)$. A typical neutron star radius being $10 \mathrm{~km}$ indicates that a Yukawa force detectable by LIGO must have range $m_{\text {med }}^{-1} \simeq \mathcal{O}(20-750 \mathrm{~km})$, i.e. $m_{\text {med }} \simeq 10^{-11}-3 \times 10^{-13} \mathrm{eV}$.

To determine the inspiral dynamics we require the total energy of the system,

$$
E_{\text {tot }}=-\frac{G_{N} \mu\left(M_{1}+M_{2}\right)}{\Delta}\left(1+\widetilde{\alpha}^{\prime} e^{-m_{\operatorname{med}} \Delta}\right)+\frac{1}{2} \mu \Delta^{2} \omega^{2} .
$$

The last term is the kinetic energy of the stars, neglecting their spin and internal structure. The power radiated via gravitational waves is $[65,66]$

$$
\frac{\mathrm{d} E_{\mathrm{GW}}}{\mathrm{d} t}=\frac{32}{5} G_{N} \mu^{2} \Delta^{4} \omega^{6} .
$$

\footnotetext{
${ }^{1}$ Note the factor $\left(1+m_{\text {med }} \Delta\right)$ was neglected in ref. [20], hence eq. (2.3) and those that follow disagree with this reference.
} 
Equating $-\mathrm{d} E_{\mathrm{GW}} / \mathrm{d} t$ to the time derivative of eq. (2.4), we obtain the rate of change of the orbital frequency,

$$
\frac{\mathrm{d} \omega}{\mathrm{d} t}=-\frac{32}{5} G_{N} \mu \Delta^{2} \omega^{5} g\left(\widetilde{\alpha}^{\prime}, m_{\mathrm{med}}, \Delta\right)
$$

where

$$
g=-\frac{3+\widetilde{\alpha}^{\prime} e^{-m_{\mathrm{med}} \Delta}\left(3+m_{\mathrm{med}} \Delta\left(3+m_{\mathrm{med}} \Delta\right)\right)}{1+\widetilde{\alpha}^{\prime} e^{-m_{\mathrm{med}} \Delta}\left(1+m_{\mathrm{med}} \Delta\left(1-m_{\mathrm{med}} \Delta\right)\right)} .
$$

In the massless mediator limit, this reduces to $g=-3$, and the classical gravity-only result is recovered [65]. In this limit the effect of the infinite range fifth-force results merely in a modification of the apparent strength of gravity, parameterized by the replacement $G_{N} \rightarrow$ $G_{N}\left(1+\widetilde{\alpha}^{\prime}\right)$ in eq. (2.3). Eq. (2.3) can then be used to eliminate $\Delta$ from eq. (2.6), yielding

$$
\frac{\mathrm{d} \omega}{\mathrm{d} t}=\frac{96}{5}\left(G_{N} \mathcal{M}_{c}\right)^{5 / 3}\left(1+\widetilde{\alpha}^{\prime}\right)^{2 / 3} \omega^{11 / 3} .
$$

We see that an infinite range Coulomb-like force results in a modified chirp mass

$$
\widetilde{\mathcal{M}}_{c} \equiv \mathcal{M}_{c}\left(1+\widetilde{\alpha}^{\prime}\right)^{2 / 5}
$$

The system is thus indistinguishable from a purely gravitationally interacting system with a different chirp mass. For non-zero $m_{\text {med }}$, eqs. (2.3) and (2.6) must be solved numerically, as there is no analytic solution for $\Delta(\omega)$ in eq. (2.3).

In figure 1, the gravitational wave frequency is shown as a function of time for three choices of $\widetilde{\alpha}^{\prime}=\{1,0.25,0.025\}$, and for two different values of $m_{\text {med }}^{-1}=\{100,300\} \mathrm{km}$. On the vertical axis, we plot $f_{\mathrm{GW}}^{-8 / 3}$ rather than just $f_{\mathrm{GW}}$ because this choice yields straight lines in the gravity only scenario (dotted black line in figure 1). In the pure gravity scenario, the unknown source distance causes the largest uncertainty in reconstructing the chirp mass. This uncertainty was estimated as $\sim 0.4 \%$ in the discovery paper of GW170817 [4]. However, the chirp mass in the detector frame has a much smaller uncertainty, $\lesssim 0.067 \%$. As a conservative choice, we show the larger uncertainty, i.e, $0.4 \%$, as the grey band in figure 1 and we use this value throughout the text. A more precise knowledge of the source distance will improve the constraints on new physics considerably.

For $m_{\text {med }}^{-1}=300 \mathrm{~km}$ and large values of $\widetilde{\alpha}^{\prime}$, we see that $\mathrm{d} \omega / \mathrm{d} t$ is a still constant, but clearly different to the gravity only solution, for the entire frequency range in which LIGO is sensitive. In other words, the Yukawa force looks like a Coulomb force to LIGO, and we would simply reconstruct the event with an alternative, incorrect, value of the chirp mass. There is thus no sensitivity to the new fifth-force for these parameters from GW signals alone; an electromagnetic signal associated with a GW event with reconstructed masses above the Chandrasekhar limit would indicate a modification induced by a dark force.

On the other hand, larger mediator masses and smaller fifth-force couplings lead to deviations that occur part-way through the time domain of the LIGO sensitivity band. These scenarios would be observed as prematurely coalescing binaries. Even for $\widetilde{\alpha}^{\prime}=0.025$ and $m_{\text {med }}^{-1}=100 \mathrm{~km}$, the coalescence time is shifted by $\sim 6 \mathrm{~s}$ relative to that predicted by gravity alone (with the chirp mass determined from the first part of the wave form). In this work, we do not consider higher order corrections to the gravitational wave emission. 


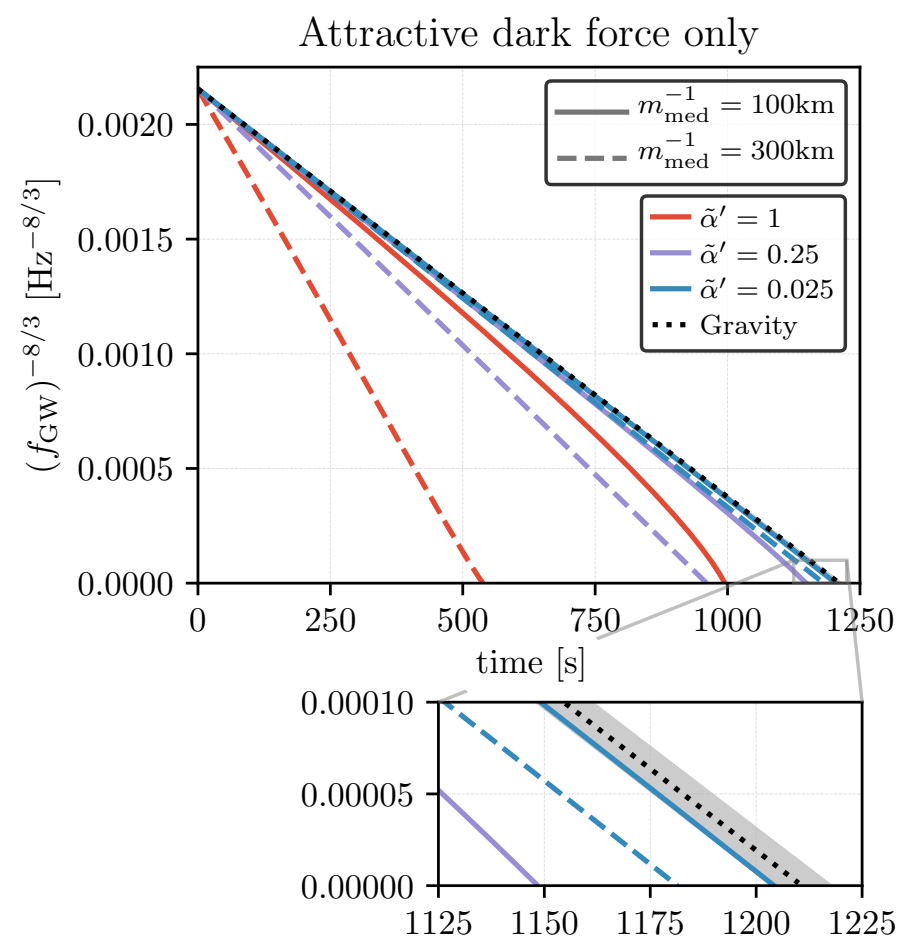

Figure 1. Gravitational wave frequency $f_{\mathrm{GW}}^{-8 / 3}(t)$ as a function of time for a neutron star binary with various values of the fifth-force parameters $\widetilde{\alpha}^{\prime}$ and $m_{\text {med }}$. In particular, we show results for $m_{\text {med }}^{-1}=\{100,300\} \mathrm{km}$ (solid, dashed lines) and three choices of $\widetilde{\alpha}^{\prime}=\{1,0.25,0.025\}$ (red, purple, and blue colored lines). We consider here the limit that the system loses energy only by gravitational wave emission, but not by radiation of light mediator particles. This situation is realized approximately if the binary partners have equal dark charge-over-mass ratios. The gray band indicates the typical error on reconstructing the chirp mass, which we conservatively take to be $0.4 \%$ based on ref. [4]. The boundary condition of the differential equation is the threshold sensitivity of LIGO, $f_{\mathrm{GW}}(0)=10 \mathrm{~Hz}$ [67]. The neutron star masses are $M_{1}=M_{2}=1.25 M_{\odot}$.

However, we note that these corrections can potentially break the degeneracy between the effects of the dark force and gravity in the case where the Yukawa potential of the dark force is unsuppressed throughout the observation window of the waveform [70]. Finally, a more accurate observable can be defined by generating the predicted waveforms including these corrections and comparing the discrepancies with and without these corrections to the instrumental noise curves.

The modification of Kepler's law, eq. (2.3), changes the initial separation of the neutron stars compared to the gravity-only case at the time when the waveform enters the LIGO frequency band. This in turn can modify the amplitude of the signal. For attractive (repulsive) dark forces one obtains an increase (decrease) in the amplitude. More concretely, the gravitational wave amplitude, $A_{\mathrm{GW}}(t)$, is given by $[20,68]$

$$
A_{\mathrm{GW}}=\frac{4 G_{N} \mu}{d_{L}} \omega^{2} \Delta^{2}
$$


where $d_{L}$ is the luminosity distance of the GW source. In the pure gravity scenario, $A_{\mathrm{GW}} \times$ $d_{L}$ is $6.3 \times 10^{-22} \mathrm{Mpc}$ at the time when the GW emission enters the LIGO frequency band. In the presence of a dark force with $\left|\widetilde{\alpha}^{\prime}\right|=0.25$ and mediator mass $m_{\text {med }}^{-1}=300 \mathrm{~km}$, the amplitude at this time becomes $6.65 \times 10^{-22} \mathrm{Mpc}$ and $5.96 \times 10^{-22} \mathrm{Mpc}$ for an attractive and repulsive dark force, respectively.

\subsection{Ultra-light boson radiation}

Another source of energy loss is radiation of the ultra-light mediator particles [11, 20]. The leading contribution to the power radiated via vector mediators in the multipole expansion is $[55,69]$

$$
\frac{\mathrm{d} E_{\text {dipole }}^{V}}{\mathrm{~d} t}=\frac{2}{3} \alpha^{\prime} \mu^{2} \gamma^{2} \omega^{4} \Delta^{2} \operatorname{Re}\left\{\sqrt{1-\left(\frac{m_{\mathrm{med}}}{\omega}\right)^{2}}\left[1+\frac{1}{2}\left(\frac{m_{\mathrm{med}}}{\omega}\right)^{2}\right]\right\},
$$

where $\gamma \equiv Q_{1} / M_{1}-Q_{2} / M_{2}$ is the difference in charge to mass ratios of the binary partners. Eq. (2.11) thus illustrates that dipole radiation is only possible if the two stars have different dark charge-over-mass ratio. For scalar mediators, the power of the dipole radiation is [55, $69]$

$$
\frac{\mathrm{d} E_{\mathrm{dipole}}^{S}}{\mathrm{~d} t}=\frac{1}{3} \alpha^{\prime} \mu^{2} \gamma^{2} \omega^{4} \Delta^{2} \operatorname{Re}\left\{\left[1-\left(\frac{m_{\text {med }}}{\omega}\right)^{2}\right]^{3 / 2}\right\} .
$$

In what follows, we will focus on vector radiation as a concrete example.

The energy loss to both gravitational wave and ultra-light boson radiation must equal the orbital energy loss

$$
\frac{\mathrm{d} E_{\mathrm{tot}}}{\mathrm{d} t}=-\left(\frac{\mathrm{d} E_{\mathrm{GW}}}{\mathrm{d} t}+\frac{\mathrm{d} E_{\text {dipole }}^{V}}{\mathrm{~d} t}\right) .
$$

In figure 2 we consider the limit where the system loses energy to dipole radiation and to gravitational waves, but gravitational wave emission is not affected by a dark force acting between the binary partners. In other words, we set $\widetilde{\alpha}^{\prime}=0$, but $\widetilde{\beta}^{\prime} \neq 0$ in eq. (2.13). This scenario corresponds to the case of only one neutron star being appreciably charged. We obtain

$$
\begin{aligned}
\frac{\mathrm{d} \omega}{\mathrm{d} t}= & \frac{96}{5}\left(G_{N} \mathcal{M}_{c}\right)^{5 / 3} \omega^{11 / 3}+\frac{1}{2} G_{N}\left(M_{1}+M_{2}\right) \widetilde{\beta}^{\prime} \omega^{3} \\
& \times \operatorname{Re}\left\{\sqrt{1-\left(\frac{m_{\text {med }}}{\omega}\right)^{2}}\left[1+\frac{1}{2}\left(\frac{m_{\text {med }}}{\omega}\right)^{2}\right]\right\},
\end{aligned}
$$

where we have defined

$$
\widetilde{\beta}^{\prime} \equiv \frac{4 \alpha^{\prime} \gamma^{2} \mu^{2}}{G_{N} M_{1} M_{2}} .
$$

$\widetilde{\beta^{\prime}}$ parameterizes the magnitude of the radiation effect relative to gravity in the same way as $\widetilde{\alpha}^{\prime}$ from eq. (2.2) parameterizes the effect of a new force between the binary partners. There is a direct correspondence $\widetilde{\alpha}^{\prime}$ and $\widetilde{\beta}^{\prime}$ when the masses of the neutron stars are the same and we take $Q_{1}=Q_{2}$ for $\widetilde{\alpha}^{\prime}$ and $Q_{2}=0$ for $\widetilde{\beta}^{\prime}$, i.e.

$$
\left.\widetilde{\alpha}^{\prime}\right|_{Q_{2}=Q_{1}}=\frac{\alpha^{\prime} Q_{1}^{2}}{G_{N} M_{1}^{2}}=\left.\widetilde{\beta}^{\prime}\right|_{Q_{2}=0} .
$$




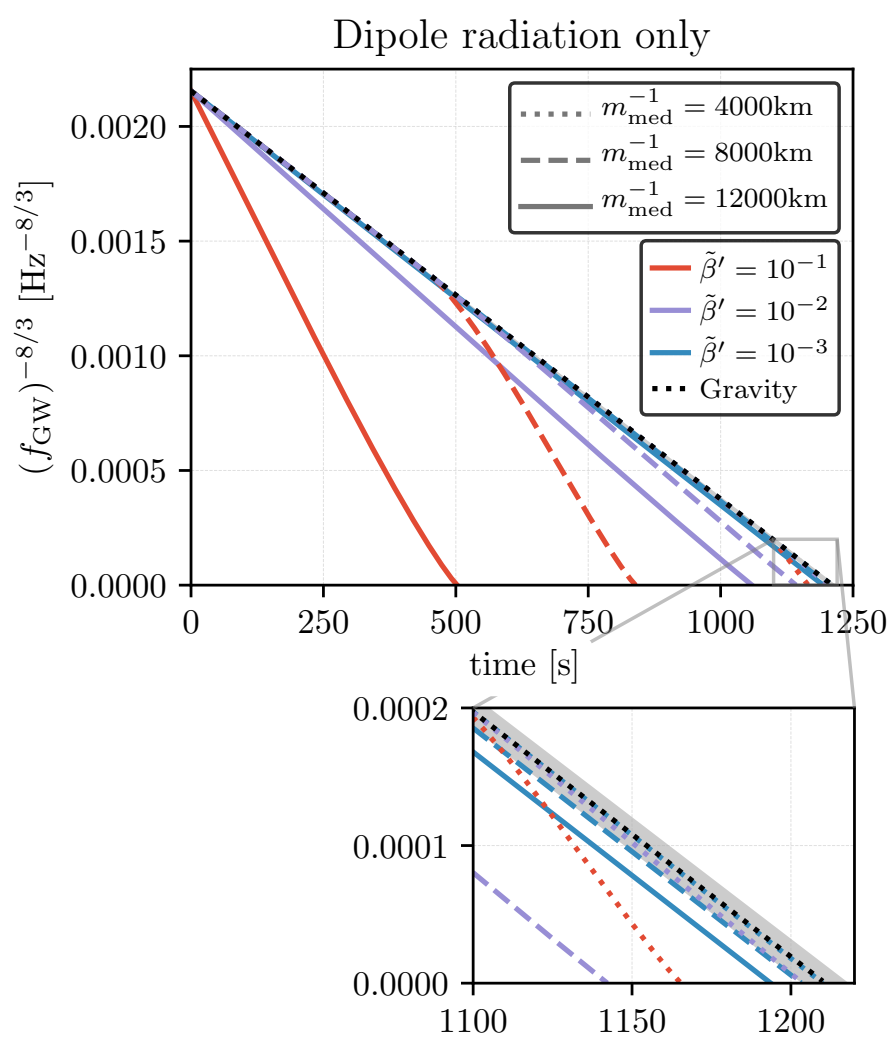

Figure 2. Gravitational wave frequency $f_{\mathrm{GW}}^{-8 / 3}(t)$ as a function of time for a neutron star binary with various values of the fifth-force parameters $\tilde{\beta}^{\prime}$ and $m_{\text {med }}$. We consider here the limit that the system loses energy via dipole radiation and gravitational wave emission, but that the latter is unaffected by a dark force acting between the binary partners. This situation is realized if one of the stars does not contain any dark sector particles. We show results for $m_{\text {med }}^{-1}=\{4000,8000,12000\} \mathrm{km}$ (dotted, dashed, solid lines) and three choices of $\widetilde{\beta}^{\prime}=\left\{10^{-1}, 10^{-2}, 10^{-3}\right\}$ (red, purple, and blue colored lines). The gray band indicates the typical error on reconstructing the chirp mass, which we take to be $0.4 \%$ based on ref. [4]. The boundary condition of the differential equation is the threshold sensitivity of LIGO, $f_{\mathrm{GW}}(0)=10 \mathrm{~Hz}$ [67]. The neutron star masses are $M_{1}=M_{2}=1.25 M_{\odot}$.

In figure 2 we show solutions of eq. (2.14) varying both $m_{\text {med }}$ and $\widetilde{\beta^{\prime}}$. The largest value of $m_{\text {med }}^{-1}$ shown, $12000 \mathrm{~km}$, is chosen such that dipole radiation is switched on while the waveform is still outside LIGO's frequency band. We see that the effect of radiation is observable even in this case, in contrast to the effect produced by a dark force with the same range, see figure 1 . The reason is the different ways in which the right hand sides of eqs. (2.8) and (2.14) scale with $\omega$. For smaller values of $m_{\text {med }}^{-1}$, the curves in figure 1 exhibit a pronounced kink around the point where $m_{\text {med }} \sim \omega$. The kink is observable for $\widetilde{\beta}^{\prime} \gtrsim 10^{-3}$, with some dependence on $m_{\text {med }}^{-1}$. This means that the sensitivity to $\widetilde{\beta}^{\prime}$ in the radiation-dominated scenario is about an order of magnitude better than the sensitivity to $\widetilde{\alpha}^{\prime}$ in the dark force-dominated scenario from section 2.1. This difference arises because the two effects arise at different order in an expansion in $\omega \Delta \sim 0.1$. 


\begin{tabular}{|ll|}
\hline Neutron star parameter & \\
\hline radius $R$ & $10 \mathrm{~km}$ \\
baryonic mass $M_{b}$ & $1.25 M_{\odot}$ \\
uniform baryonic density $\rho_{b}$ & $3.3 \times 10^{38} \mathrm{GeV} \mathrm{cm}^{-3}$ \\
age $t_{\mathrm{NS}}$ & $7 \mathrm{Gyr}$ \\
escape velocity $v_{\mathrm{esc}}(R)$ & $1.8 \times 10^{5} \mathrm{kms}^{-1}$ \\
number of neutrons $N_{B}$ & $1.48 \times 10^{57}$ \\
temperature $T_{\mathrm{NS}}$ & $10^{5} \mathrm{~K}$ \\
\hline
\end{tabular}

Table 1. The parameters of the benchmark neutron stars used in our numerical estimates. These parameter choices are representative of the recently observed neutron star binary merger [4].

The upshot of this section is that for neutron star binaries in which both stars carry a dark charge and have similar dark charge-to-mass ratio, LIGO may be able to establish the existence of a new long-range force if $\widetilde{\alpha}^{\prime} \gtrsim \mathcal{O}(0.01)$ and $50 \mathrm{~km} \lesssim m_{\text {med }}^{-1} \lesssim 300 \mathrm{~km}$. In binary systems with large dark charge-to-mass ratio difference $\gamma$, observable effects from dipole radiation of the new force carriers can be expected for $\widetilde{\beta}^{\prime} \gtrsim \mathcal{O}\left(10^{-3}\right)$ and $m_{\text {med }}^{-1} \gtrsim \mathcal{O}(4000 \mathrm{~km})$. In this case, there is no upper limit on the observable values of $m_{\text {med }}^{-1}$.

Of course, radiation of dark force into higher multipoles is possible even for systems that are symmetric in their charge-to-mass ratio. However, such radiation is suppressed by additional powers of $\omega \Delta$, so we do not expect it to dominate over the effect of the new longrange force between the binary partners. Moreover, the effect of quadrupole radiation, like the effect of the dark force, would be degenerate with the effect of gravity at large $m_{\text {med }}^{-1}$, making it unobservable in this regime independent of the suppression by $\omega \Delta$. At smaller $m_{\text {med }}^{-1}$, however, radiation into higher multipoles would lead to the same kinks as dipole radiation (see figure 2), offering an extra handle for establishing the radiation effect. The kinks occur within the LIGO sensitivity window for $4000 \mathrm{~km}<m_{\text {med }}^{-1}<12000 \mathrm{~km}$. In the following sections we will investigate the viability of the parameter values indicated above.

We note that the reach in $m_{\text {med }}^{-1}$ may be significantly enhanced in the future by combining LIGO/VIRGO measurements with results from observatories which are sensitive to lower gravitational wave frequencies [70]. These observatories will be able to observe neutron star binary systems long before the merger. If the separation of the binary partners is larger than $m_{\text {med }}^{-1}$ while the system is observable by the lower frequency detectors, but below $m_{\text {med }}^{-1}$ when it enters the LIGO/VIRGO frequency band, the effect of the dark force can be observed in a combined analysis.

To end this section we remark that while the above discussion has been in the context of neutron stars these results are also applicable to black hole or mixed binaries so long as the black holes can carry the appropriate charge.

\section{Constraints on repulsive forces}

We now focus our attention on the case of a repulsive force between the dark particles captured by binary neutron stars. Our results on this scenario are summarized in figure 4 . 

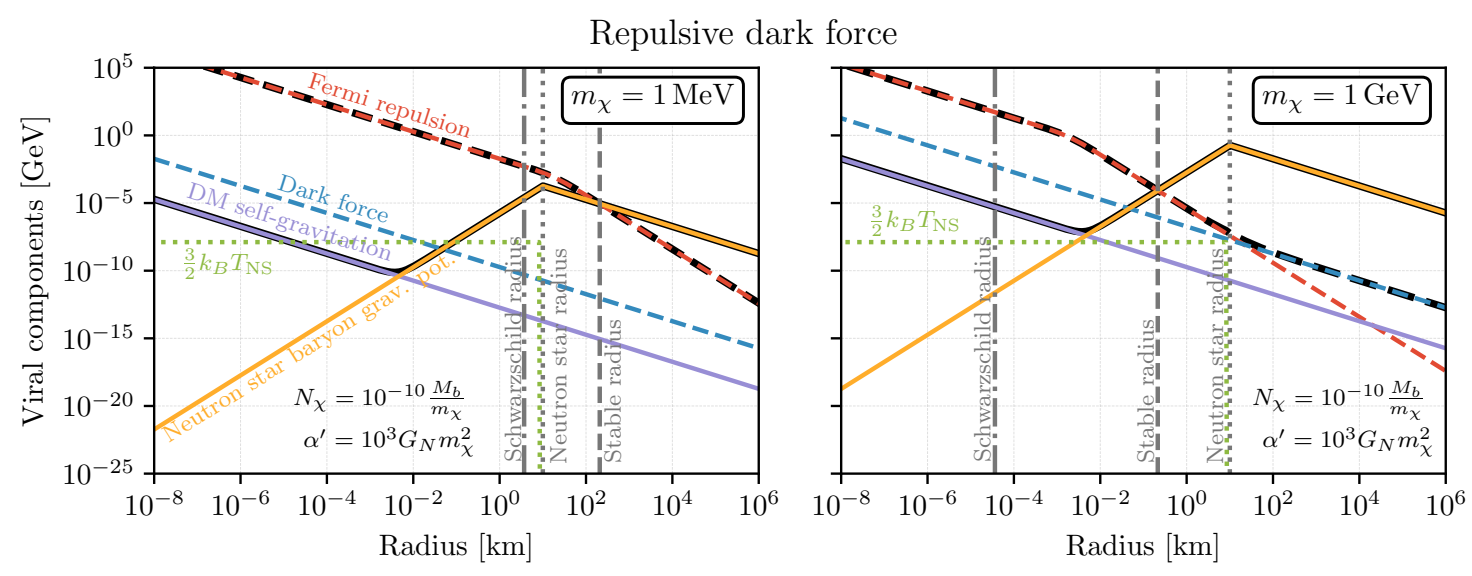

Figure 3. Visualization of the different contributions to the virial equation, eq. (3.10), as a function of the radius for two different choices of the DM mass. We plot the absolute values of the respective contributions, and indicate attractive (repulsive) forces as solid (dashed) lines. The black solid (black dashed) line correspond to the sum of all attractive (repulsive) forces. The solution to eq. (3.10), i.e. the intersection of the black solid and black dashed lines, is highlighted by a vertical dashed line.

The four panels of this figure show, for different assumptions on the particle mass $m_{\chi}$, the limits on the dark fine structure constant $\alpha^{\prime}$ and the total mass of all $\chi$ particles in the neutron star, $M_{\chi}$. We normalize $\alpha^{\prime}$ to the effective strength of gravity, $G_{N} m_{\chi}^{2}$, and $M_{\chi}$ to the mass of the baryons in the neutron star, $M_{b}$. In the following, we discuss the various constraints shown in figure 4 one by one.

Throughout our discussion of constraints we shall quote bounds on the dark force parameter $\widetilde{\alpha}^{\prime}$ under the assumption that both neutron stars have identical dark charges and masses. In the opposite limit that one neutron star carries a dark charge while the other does not, equal constraints apply on the parameter $\widetilde{\beta}^{\prime}$. It is crucial here that we have defined $\widetilde{\beta}^{\prime}$ such that under the assumption that only one neutron star is charged its value is equal to that of $\widetilde{\alpha}^{\prime}$ for equally charged neutron stars.

\subsection{Binding potential for DM particles}

It is intuitively clear that a repulsive force between dark particles will limit the maximum number of such particles that can accumulate in or around a neutron star or black hole. Irrespective of the capture or production mechanism, the number of particles is saturated once the net potential of the compact object ceases to be attractive. Consider the potential $V(r)$ felt by a single DM particle $\chi$ of charge $q_{\chi}$ and mass $m_{\chi}$ at a distance $r$ from the center of the star:

$$
V(r)=-\frac{G_{N} M m_{\chi}}{r}+\frac{\alpha^{\prime} q_{\chi} Q e^{-m_{\mathrm{med}} r}}{r} .
$$

Here, $Q$ is the total dark charge of the star, and

$$
M=M_{b}+M_{\chi}
$$


is its total mass (including the total baryonic mass, $M_{b}$, and the total mass of dark particles, $\left.M_{\chi}\right) . M_{\chi}$ can be traded for the number of particles, $N_{\chi}$, of a given mass $m_{\chi}$ :

$$
M_{\chi}=m_{\chi} N_{\chi} .
$$

Insisting that the potential is attractive $(V(r) \leq 0)$ at length scales $r \ll m_{\text {med }}^{-1}$ (where the Yukawa force is effectively Coulomb-like) yields the condition

$$
N_{\chi} \lesssim \frac{M_{b} m_{\chi}}{\alpha^{\prime} q_{\chi}^{2} / G_{N}-m_{\chi}^{2}}
$$

This constraint is shown in light blue in figure 4.

We can use eq. (3.4) to derive a constraint on the effective strength $\widetilde{\alpha}^{\prime}$ of the new force relative to gravity. Plugging eq. (3.4) into eq. (2.2), we obtain

$$
\widetilde{\alpha}^{\prime} \lesssim \frac{G_{N} m_{\chi}^{2}}{\alpha^{\prime} q_{\chi}^{2}}
$$

\subsection{Size of the dark core}

In order for the effect of the dark force on the neutron star to be predictable using the calculations of section 2, it is necessary that the core of dark sector particles be significantly smaller than the screening radius of the force. Otherwise, the interactions of the neutron stars will be primarily with a cloud of dark particles in which they are both embedded, rather than remaining a simple central-force problem. This would require a much more sophisticated treatment. We leave the dynamics of such a system for future work, and here aim to enforce the requirement that the dark particle core be smaller in extent than the screening radius $m_{\text {med }}^{-1}$.

We remark however that the far-outlying particles at the edge of the neutron stars' dark matter halo will be very easily stripped off in the presence of the second neutron star because they feel the dark force field created by that star's DM halo long before the inspiral dynamics can be affected. Thus, those outlying particles would not affect the gravitational wave signal, as they would no longer be bound to just one of the two stars.

Using the virial theorem, we can calculate the stable core radius for given $\alpha^{\prime}, m_{\chi}$, and $N_{\chi}$. The general form of the virial theorem (valid for non-relativistic and relativistic systems) is $\left\langle\sum_{i} \mathbf{p}_{i} \cdot \mathbf{v}_{i}\right\rangle=-\left\langle\sum_{i} \mathbf{F}_{i} \cdot \mathbf{r}_{i}\right\rangle$ [71], where $i$ runs over all particles, and $\mathbf{r}_{i}, \mathbf{p}_{i}$, $\mathbf{F}_{i}$ are their positions, their momenta, and the forces they experience, respectively. The notation $\langle\ldots\rangle$ indicates time averaging, which here translates to the requirement that the system is in a stable configuration. For an isotropic potential, the virial equation in its relativistic form simplifies to $[72,73]$

$$
\left\langle\sum_{i} \frac{\mathbf{p}_{i}^{2}}{\sqrt{\mathbf{p}_{i}^{2}+m_{\chi}^{2}}}\right\rangle=\left\langle\sum_{i} r_{i} \frac{\partial V_{i}\left(r_{i}\right)}{\partial r_{i}}\right\rangle .
$$

The potential energy for a single particle at the edge of the dark core (radius $R_{\chi}$ ) is given by

$$
V\left(R_{\chi}\right)=-\frac{\left(G_{N} m_{\chi}^{2}-\alpha^{\prime}\right) N_{\chi}}{R_{\chi}}-G_{N} M_{b} m_{\chi}\left\{\begin{array}{ll}
\left(3 R^{2}-R_{\chi}^{2}\right) /\left(2 R^{3}\right) & R_{\chi} \leq R \\
1 / R_{\chi} & R_{\chi}>R
\end{array} .\right.
$$




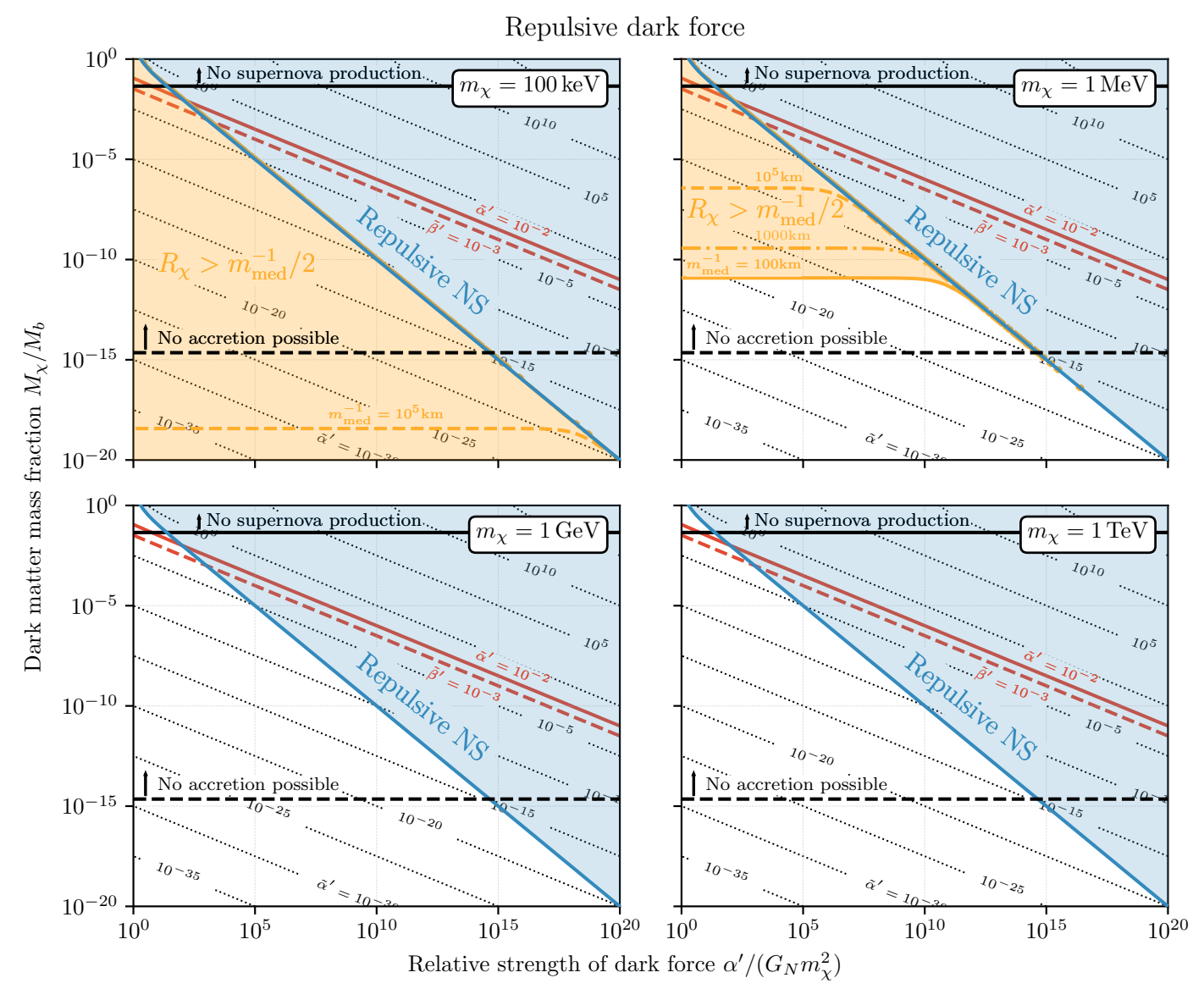

Figure 4. Constraints on the coupling $\alpha^{\prime}$ of a repulsive dark force as a function of the total mass $M_{\chi}$ of dark particles bound to the neutron star. We normalize $\alpha^{\prime}$ to the strength of gravity, $G_{N} m_{\chi}^{2}$, and $M_{\chi}$ to the baryonic mass of the neutron star, $M_{b}$. The four panels correspond to different choices of $m_{\chi}$, as indicated in the plots. The dotted contours show the value of $\widetilde{\alpha}^{\prime}$ (in the case of neutron stars with equal dark charge-over-mass ratio) or $\widetilde{\beta}^{\prime}$ (for the case that only one neutron star carries a dark charge, see eq. (2.2)) at each point in the plot. We indicate in red the estimated minimum value of $\widetilde{\alpha}^{\prime}$ or $\widetilde{\beta}^{\prime}$ required for the dark force to leave an observable imprint in LIGO data. Regions of parameter space where the neutron star no longer has an overall attractive potential for DM, i.e., $N_{\chi}$ exceeds the bound of eq. (3.4), are shaded in blue. Note that this bound is independent of the DM production or capture mechanism. Bounds shown in black are model-dependent: in the region above the black-dashed line, the DM population inside the neutron cannot be generated by capture from the halo, based on the geometric arguments that lead to eq. (3.12). Above the black solid line, the hypothesis that the DM population is produced during the supernova that created the neutron star is not tenable because the energy available in a supernova (as measured in SN 1987A) is not sufficient. Inside the yellow shaded region, the dark core has a radius larger than the range of new Yukawa force, $R_{\chi} \geq m_{\text {med }}^{-1} / 2$. The solid, dot-dashed, and dashed yellow lines correspond to $m_{\text {med }}^{-1}=\left\{100 \mathrm{~km}, 1000 \mathrm{~km}, 1 \times 10^{5} \mathrm{~km}\right\}$, respectively. Within the yellow regions, the DM particles would be stripped away from their host neutron star before they can have a significant impact on the inspiral. The neutron star parameters are chosen in accordance with table 1 , and we normalize $\alpha^{\prime}$ such that $q_{\chi}=1$. 
The first term is the combination of the gravitational self-interactions of the exotic particles and the new dark interactions in the zero screening limit $R_{\chi} m_{\text {med }} \ll 1$. The second term is the gravitational interaction between the baryons and the test particle defined separately in the two cases where the $\chi$-core is either smaller or larger than the radius $R$ of the baryonic matter. We assume that the dominant contribution to the average momentum on the left hand side of eq. (3.6) arises from Pauli repulsion. (We will justify this assumption shortly.) The $\mathbf{p}_{i}$ are then of the order of the Fermi momentum of a degenerate 3-dimensional system of free spin- $1 / 2$ fermions (with no additional internal degrees of freedom)

$$
p_{F}=\left(\frac{3 \pi^{2} N_{\chi}}{V}\right)^{1 / 3}=\frac{1}{R_{\chi}}\left(\frac{9 \pi N_{\chi}}{4}\right)^{1 / 3}
$$

where $V$ is the volume of the $\chi$-core. For repulsive forces, we find that the system is always in a non-relativistic configuration in the parameter regions of interest to us (unshaded portions of figure 4), allowing the simplification

$$
\frac{p_{F}^{2}}{\sqrt{p_{F}^{2}+m_{\chi}^{2}}}=\frac{1}{m_{\chi} R_{\chi}^{2}}\left(\frac{9 \pi N_{\chi}}{4}\right)^{2 / 3} .
$$

Inserting eq. (3.9) and eq. (3.7) into the virial theorem, eq. (3.6), yields

$$
\frac{1}{m_{\chi} R_{\chi}^{2}}\left(\frac{9 \pi N_{\chi}}{4}\right)^{2 / 3}=\frac{\left(G_{N} m_{\chi}^{2}-\alpha^{\prime}\right) N_{\chi}}{R_{\chi}}+G_{N} M_{b} m_{\chi}\left\{\begin{array}{ll}
R_{\chi} / R^{3} & R_{\chi} \leq R \\
1 / R_{\chi}^{2} & R_{\chi}>R
\end{array} .\right.
$$

The different contributions to this equation are represented graphically in figure 3 , where dashed lines indicate repulsive forces and solid lines indicate attractive forces. The plots also show the contribution from ordinary thermal pressure, which, however, is relevant only for relatively heavy DM $\left(m_{\chi} \gtrsim \mathrm{TeV}\right)$, weak dark forces $\left(\alpha^{\prime} \sim G_{N} m_{\chi}^{2}\right)$, and small dark cores $\left(M_{\chi} \lesssim 10^{-20} M_{b}\right)$. This parameter region is not of interest to us as it corresponds to tiny $\widetilde{\alpha}^{\prime}$; this justifies our previous assumption that degeneracy pressure dominates. The intersection between the sum of all repulsive contributions and the sum of all attractive contributions corresponds to the solution of eq. (3.10) and thus gives the radius of the dark core as a function of $m_{\chi}, \alpha^{\prime}$, and $N_{\chi}$.

For light DM mass, Fermi-repulsion results in radii larger than the size of the neutron star. The regions of parameter space where $R_{\chi}>m_{\text {med }}^{-1} / 2$ are shaded in yellow in figure 4 . A $\chi$-core with such an extent results in inspiral dynamics very different to those assumed in our estimates so far. In particular, as argued above, particles at radii larger than $m_{\text {med }}^{-1}$ will be efficiently stripped from the neutron star. The yellow curves, therefore, can be taken as an indication of the maximum amount of charge that fits geometrically within the domain of influence of a single neutron star, and thus can contribute to a dark-force-induced change in inspiral dynamics and gravitational wave signals.

\subsection{Constraints on dark core production via particle accretion}

There exists an additional upper bound on $N_{\chi}$ under the assumption that the particles giving the neutron star its dark charge have been accreted from the host galaxy's DM 
halo. An upper limit on the number $N_{\chi}^{\text {capt }}$ of DM particles $(\chi)$ accreted can be obtained by assuming that any DM particle that passes through the neutron star over its lifetime $t_{\mathrm{NS}}$ is captured. (A more realistic estimate based on the dynamics of baryon- $\chi$ scattering is discussed in appendix A.) The upper limit on $N_{\chi}^{\text {capt }}$ is based on the effective geometric cross section of the neutron star, $\sigma_{\text {geom }}^{\text {eff }}$ given by

$$
\sigma_{\text {geom }}^{\text {eff }} \equiv \sigma_{\text {geom }}\left(1+\frac{v_{\text {esc }}^{2}}{\bar{v}^{2}}\right) \simeq \frac{\sigma_{\text {geom }} v_{\text {esc }}^{2}}{\bar{v}^{2}}
$$

where $v_{\text {esc }}$ is the escape velocity at the surface of the neutron star and $\bar{v}$ is the average $\chi$ velocity relative to the neutron star at infinity. The velocity-dependent term by which $\sigma_{\text {geom }}^{\text {eff }}$ differs from the geometric cross section $\sigma_{\text {geom }}=\pi R^{2}$ accounts for the focusing of the DM wind by the neutron star's gravitational field [74]. In the second equality in eq. (3.11), we use $v_{\text {esc }}>\bar{v}$, an approximation that is well satisfied for typical galactic DM velocities $\bar{v} \sim 200 \mathrm{~km} \mathrm{~s}^{-1}$ and escape velocities at the surface of a neutron star $v_{\mathrm{esc}} \sim 100000 \mathrm{~km} \mathrm{~s}^{-1}$. The upper limit on $N_{\chi}^{\text {capt }}$ is then

$$
N_{\chi}^{\mathrm{capt}} \leq \frac{\pi R^{2} v_{\mathrm{esc}}^{2} t_{\mathrm{NS}} \rho_{\chi}}{\bar{v} m_{\chi}}
$$

where $\rho_{\chi}$ is the mass density of $\chi$ in the vicinity of the neutron star. This constraint is shown as a horizontal black dashed line in figure 4 .

As the constraints on $N_{\chi}$ from eqs. (3.4) and (3.12) scale inversely to one another with respect to $m_{\chi}$, the value $m_{\chi}^{\text {opt }}$ that maximizes $N_{\chi}$ and thus $\widetilde{\alpha}^{\prime}$ occurs when both constraints are equal. We find

$$
\left(m_{\chi}^{\mathrm{opt}}\right)^{2}=\frac{\pi R^{2} v_{\mathrm{esc}}^{2} t_{\mathrm{NS}} \rho_{\chi} \alpha^{\prime} q_{\chi}^{2}}{G_{N}\left(M_{b} \bar{v}+\pi R^{2} v_{\mathrm{esc}}^{2} t_{\mathrm{NS}} \rho_{\chi}\right)}
$$

and, by plugging $m_{\chi}^{\text {opt }}$ into eq. (3.5),

$$
\widetilde{\alpha}^{\prime} \leq \frac{\pi R^{2} v_{\mathrm{esc}}^{2} t_{\mathrm{NS}} \rho_{\chi}}{M_{b} \bar{v}+\pi R^{2} v_{\mathrm{esc}}^{2} t_{\mathrm{NS}} \rho_{\chi}} \simeq \frac{\pi R^{2} v_{\mathrm{esc}}^{2} t_{\mathrm{NS}} \rho_{\chi}}{M_{b} \bar{v}}=2.25 \times 10^{-15} .
$$

The second equality follows because the mass of the accreted DM particles is much smaller than the baryonic mass $M_{b}$. For the numerical estimate in the second line of eq. (3.14), we have used the neutron star parameters given in table 1, we have (very conservatively) assumed that $\rho_{\chi}$ saturates the observed DM density in the solar neighborhood $\rho_{\chi}=0.3 \mathrm{GeV} / \mathrm{cm}^{3}$, and we have taken $\bar{v}=220 \mathrm{~km} \mathrm{~s}^{-1}$. We note that this limit on $\widetilde{\alpha}^{\prime}$ is independent of both $m_{\chi}$ and $\alpha^{\prime}$. The limit is relaxed in regions of very low DM velocity $\bar{v}$.

\subsection{Constraints on dark core production in supernovae}

The upper limit on $N_{\chi}$ from eq. (3.12), and the resulting limit on $\widetilde{\alpha}^{\prime}$, eq. (3.14), were based on the assumption that the DM population bound to the neutron star arises from capture of DM particles from the halo. Relaxing this assumption and invoking more speculative production mechanisms can lead to significant boosts in the allowed values of $\widetilde{\alpha}^{\prime}$. We first note that the largest $\widetilde{\alpha}^{\prime}$ values compatible with the constraints of section 3.1 (blue regions 
in figure 4) occur in the region of parameter space where the dark force is not much stronger than gravity and where $M_{\chi} \sim M_{b}$.

One possibility for producing a massive dark core without accreting DM from the halo arises if DM particles are produced via bremsstrahlung in a new-born hot neutron star [22, 23]. The upper limit on the total mass of exotic particles produced this way arises from the measured neutrino burst of SN 1987A [75-79]. Adopting the 'Raffelt criterion' [80] that $\chi$ production should not reduce the duration of the neutrino burst from SN 1987A by more than $50 \%$, we require that the instantaneous $\chi$ luminosity should be $L_{\chi} \leq 3 \times 10^{52} \mathrm{ergs} \mathrm{s}^{-1}$. Various different effects need to be taken into account to evaluate $L_{\chi}$ [81-85]. A near future detection of supernova neutrinos in all flavors will robustly strengthen these bounds [86-88].

We conservatively estimate that the total available energy to produce $\chi$ during the supernova is $10^{53} \mathrm{ergs}$, which, under the further conservative assumption that these particles are produced at rest, translates to $M_{\chi} \lesssim 0.045 M_{\odot}$. This constraint is very weak due to the current scarcity of supernova neutrino measurements, and corresponds to approximately $1 / 3$ of the total energy of the supernova being used to produce DM. This constraint is shown as a solid black horizontal line in figure 4 . We can turn it into a constraint on $\widetilde{\alpha}^{\prime}$ by requiring that it is saturated simultaneously with the upper bound on $N_{\chi}$ from eq. (3.4) (blue shaded region in figure 4). The resulting equation can be solved for $\alpha^{\prime}$, and the result can be plugged into the definition of $\widetilde{\alpha}^{\prime}$, eq. (2.2). If we assume that the two neutron stars have similar mass and dark charge, we obtain the $m_{\chi}$-independent limit

$$
\widetilde{\alpha}^{\prime} \leq 4 \times 10^{-2}
$$

Note that the known thermal mechanisms to generate exotic particles in supernovae are inefficient for particle masses $\gtrsim 10 \mathrm{MeV}$. In figure 4 , we nevertheless plot the supernova constraint for $m_{\chi} \gtrsim 10 \mathrm{MeV}$ as an absolute upper limit on the amount of energy available to produce exotic particles in the supernova. We are, however, not aware of any mechanism that could possibly be efficient in converting an $\mathcal{O}(30 \%)$ fraction of the supernova energy into heavy particles. Any supernova-driven mechanism for DM production would lead to all neutron stars being similarly charged, and thus the LIGO sensitivity to these mechanisms is best modeled in figure 1, as the charge-to-mass ratios of the binary stars will be similar.

\subsection{Constraints on dark core production via neutron decay}

An alternative production mechanism for DM particles inside a neutron star has been proposed in ref. [89] and further investigated in ref. [90]. In this scenario, $\chi$ is abundantly produced inside the neutron star via a hypothetical exotic neutron decay mode. In this case a repulsive dark force would have an additional benefit: without it, the neutron star equation of state (EOS) would be softened because of decreased Fermi repulsion in the nuclear matter. This would preclude the existence of high-mass neutron stars with $M \simeq 2 M_{\odot}$, in conflict with observations [91, 92]. A repulsive dark force, however, stiffens the equation of state again. This places a lower bound on the size of the dark fine structure constant $\alpha^{\prime}$. Ref. [90] found a bound on the strength of the needed repulsive force as a 
function of the mediator mass. In our notation, this constraint reads

$$
\alpha^{\prime} \geq 1.5 \times 10^{-40}\left(\frac{m_{\mathrm{med}}}{100 \mathrm{~km}}\right)^{2} .
$$

Note, however, that the calculations of ref. [90] were performed in a scenario with a partially screened dark force, whereas scenarios in which the dark force could significantly impact inspiral dynamics and gravitational wave emission would require it to be unscreened inside the neutron star. This will weaken the constraint on $\alpha^{\prime}$ from EOS arguments compared to eq. (3.16). Moreover, the neutron decay scenario requires careful tuning of $m_{\chi}$ : stability of ${ }^{9}$ Be places an upper bound on the mass splitting between $\chi$ and the neutron of $1.59 \mathrm{MeV}[93$, 94], and the lifetime of ${ }^{11} \mathrm{Be}$ further strengthens this bound to require splittings of less than $0.50 \mathrm{MeV}[95]$.

To estimate the maximum quantity of hidden sector particles that can be produced via neutron decay, we consider the chemical potentials of neutrons and DM. We describe the neutrons by a Fermi gas with chemical potential

$$
\mu_{B}=\sqrt{m_{n}^{2}+\left(3 \pi^{2} n_{n}\right)^{2 / 3}}-E_{B},
$$

where $n_{n}$ is the number density of neutrons (which decreases over time in this scenario), and $E_{B}$ is a typical nuclear binding energy, taken here to be $E_{B}=9 \mathrm{MeV}$ [90]. The second term under the square root corresponds to the square of the neutron Fermi momentum.

We assume the $\chi$ particles experience an unscreened potential, i.e., $m_{\text {med }}^{-1} \gg R$, resulting in the chemical potential

$$
\mu_{\chi} \simeq m_{\chi}+\frac{\alpha^{\prime} N_{\chi}}{R_{\chi}}
$$

Neutron decays occur until $\mu_{\chi}=\mu_{B}$. Therefore, the maximum $N_{\chi}$ can be determined by equating the two chemical potentials. Choosing for $m_{\chi}$ the smallest value allowed by the lifetime of ${ }^{11} \mathrm{Be}, m_{\chi}=939.06 \mathrm{MeV}$ [95], and for $\alpha^{\prime}$ the smallest value which can still be comparable in its effects to those of gravity, $\alpha^{\prime}=G_{N} m_{\chi}^{2}$, we obtain $M_{\chi} / M_{b} \leq 0.36$. Here, we have used the neutron star parameters given in table 1, and we have assumed the radius of the dark core to be similar to the radius of the neutron star.

The above constraint on $M_{\chi} / M_{b}$ corresponds to $\widetilde{\alpha}^{\prime}=0.071$, which would be marginally detectable in LIGO/VIRGO data, see figure 1. Such a modification could be more clearly observable by comparing LIGO/VIRGO measurements with results from other lower frequency instruments [70]. Note that the upper bound on $\widetilde{\alpha}^{\prime}$ in the neutron decay scenario has been calculated for the choices of $m_{\chi}, \alpha^{\prime}$ which maximize the potential effect on inspirals; either increasing $m_{\chi}$ or increasing $\alpha^{\prime}$ leads to a tighter upper bound on the mass ratio $M_{\chi} / M_{b}$. An increased $\alpha^{\prime}$ thus leads to a decrease in the dark charge, so that overall the strength of the dark force between the binary stars is decreased. Note also that, in scenarios where dark particles are produced mainly via exotic neutron decay, there is no way to create an uncharged neutron star, so the dominant effect of the dark force is always a modification of the potential in which the neutron stars orbit as shown in figure 1, never dipole radiation of the force mediator. 
We finally note that the coupling that induces decays of the form $n \rightarrow \chi+X$ (where $X$ can, but does not have to be, the dark force mediator $A^{\prime}$ ) will always lead to a coupling of neutrons to $A^{\prime}$ at 1-loop level. This shows that the assumption of a dark force coupling only to hidden sector particles must be relaxed to produce an observable effect in the inspiral waveform. Then, however, new physics constraints from modified gravitational wave signals need to be discussed in the context of constraints arising from more generic fifth-force searches [24-26]. The interplay between these various constraints is model-dependent and would require dedicated studies.

\section{Constraints on attractive forces}

We now switch gears and consider attractive dark sector forces. In this case, important constraints arise from black hole formation inside neutron stars. Additionally, the gravitational binding of DM particles to the neutron star is now small compared to their attractive selfinteractions, leading to the possibility that the dark core can migrate out of the neutron star and stop affecting the observed inspiral dynamics. In the following we provide analytical estimates of these constraints and then present the full numerical results in figures 5 and 6 .

\subsection{Black hole formation}

As a neutron star accumulates a large number of DM particles close to its center, it runs the risk of the dense dark core collapsing into a black hole which eventually consumes the whole star $[38,45]$. The mere existence of old neutron stars tells us that black hole formation cannot be too efficient, and this in turn restricts the DM parameter space [96, 97]. The most optimistic scenario (i.e. the one in which black hole formation is least likely) assumes that the DM particle $\chi$ is a fermion. In this case, Pauli repulsion helps to stabilize the dark core at radii larger than the Schwarzschild radius.

For the following estimate we follow refs. [35, 38, 45], but assuming that the range of the dark force is always larger than the radius $R_{\chi}$ of the $\chi$ core. Using the virial theorem (see section 3.2), we determine if a stable radius exists for given values of $\alpha^{\prime}, m_{\chi}$, and $N_{\chi}$. In contrast to the case of repulsive forces, it is possible for the DM particles to be either non-relativistic, fully relativistic, or in between. Inserting the Fermi momentum $p_{F}=\left(9 \pi N_{\chi} / 4\right)^{1 / 3} / R_{\chi}$ from eq. (3.8), and the potential energy given by eq. (3.7) with the replacement $\alpha^{\prime} \rightarrow-\alpha^{\prime}$, into the virial equation eq. (3.6), we obtain

$$
\frac{1}{R_{\chi}} \frac{\left(\frac{9}{4} \pi N_{\chi}\right)^{2 / 3}}{\sqrt{\left(\frac{9}{4} \pi N_{\chi}\right)^{2 / 3}+m_{\chi}^{2} R_{\chi}^{2}}}=\frac{\left(G_{N} m_{\chi}^{2}+\alpha^{\prime}\right) N_{\chi}}{R_{\chi}}+G_{N} M_{b} m_{\chi}\left\{\begin{array}{ll}
R_{\chi} / R^{3} & R_{\chi} \leq R \\
1 / R_{\chi}^{2} & R_{\chi}>R
\end{array} .\right.
$$

This expression is of course fully analogous to eq. (3.10), and the different terms in it can be visualized in figure 3, except that now the dark force is attractive, i.e. the cyan lines should now be solid rather than dashed. If, for a given $N_{\chi}$, a solution for $R_{\chi}$ exists, the dark core is stable. If no solution exists, the core will collapse into a black hole. We have checked that in the parameter regions of interest to us, the solution for $R_{\chi}$, if it exists, is always larger than the Schwarzschild radius. 


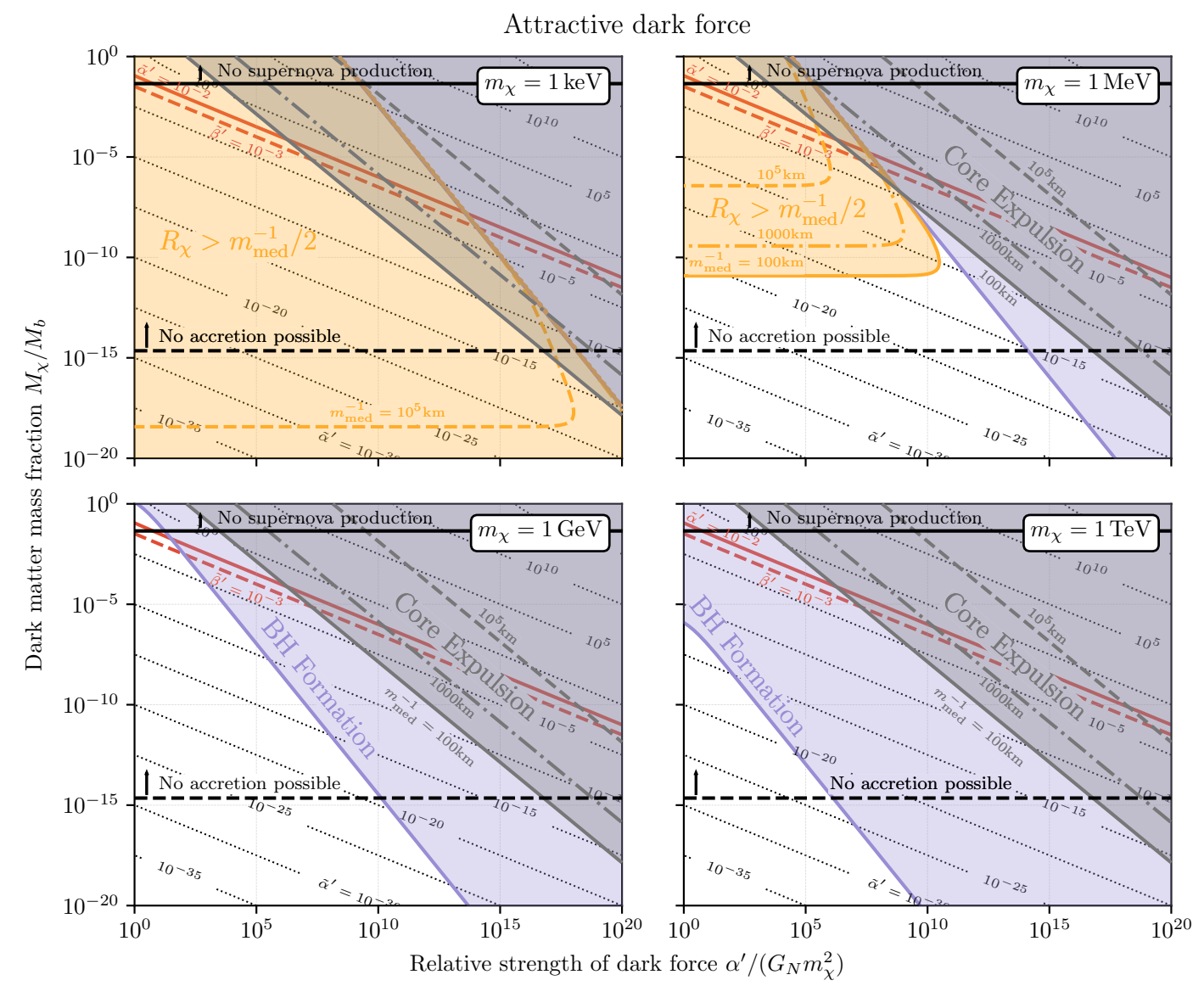

Figure 5. Constraints on an attractive dark force from binary neutron star systems as a function of the dark fine structure constant $\alpha^{\prime}$ (expressed here relative to the gravitational force) and the amount of DM bound to the neutron stars (assuming both stars carry the same amount of DM). The four panels correspond to different choices of the DM mass $m_{\chi}$, as indicated in the plots. The dotted contours show the value of $\widetilde{\alpha}^{\prime}$ (in the case of neutron stars with equal dark charge-over-mass ratio) or $\widetilde{\beta}^{\prime}$ (for the case that only one neutron star carries a dark charge, see eq. (2.2)) at each point in the plot. We indicate in red the estimated minimum value of $\widetilde{\alpha}^{\prime}$ or $\widetilde{\beta}^{\prime}$ required for the dark force to leave an observable imprint in LIGO data. In the purple region, the dark force induces collapse of the neutron stars' dark cores to black holes. Inside the gray shaded region, the dark cores are expelled from the neutron stars as soon as the screening of the force is lifted. We show this region for $m_{\text {med }}^{-1}=$ $100 \mathrm{~km}$ (solid), $1000 \mathrm{~km}$ (dot-dashed), and $1 \times 10^{5} \mathrm{~km}$. Bounds shown in black are model-dependent: in the region above the black-dashed line, the DM population inside the neutron cannot be generated by capture from the halo, based on the geometric arguments that lead to eq. (3.12). Above the solid black line, the hypothesis that the DM population is produced radiatively during the supernova that created the neutron star is not tenable because the energy available in a supernova (as measured in SN 1987A) is not sufficient. Within the yellow regions, the DM particles would be stripped away from their host neutron star before they can have a significant impact on the inspiral. The benchmark values of $m_{\text {med }}^{-1}$ are the same as for the gray DM expulsion bound. The neutron star parameters are chosen in accordance with table 1 , and without loss of generality we have chosen $q_{\chi}=1$. 
Attractive dark force

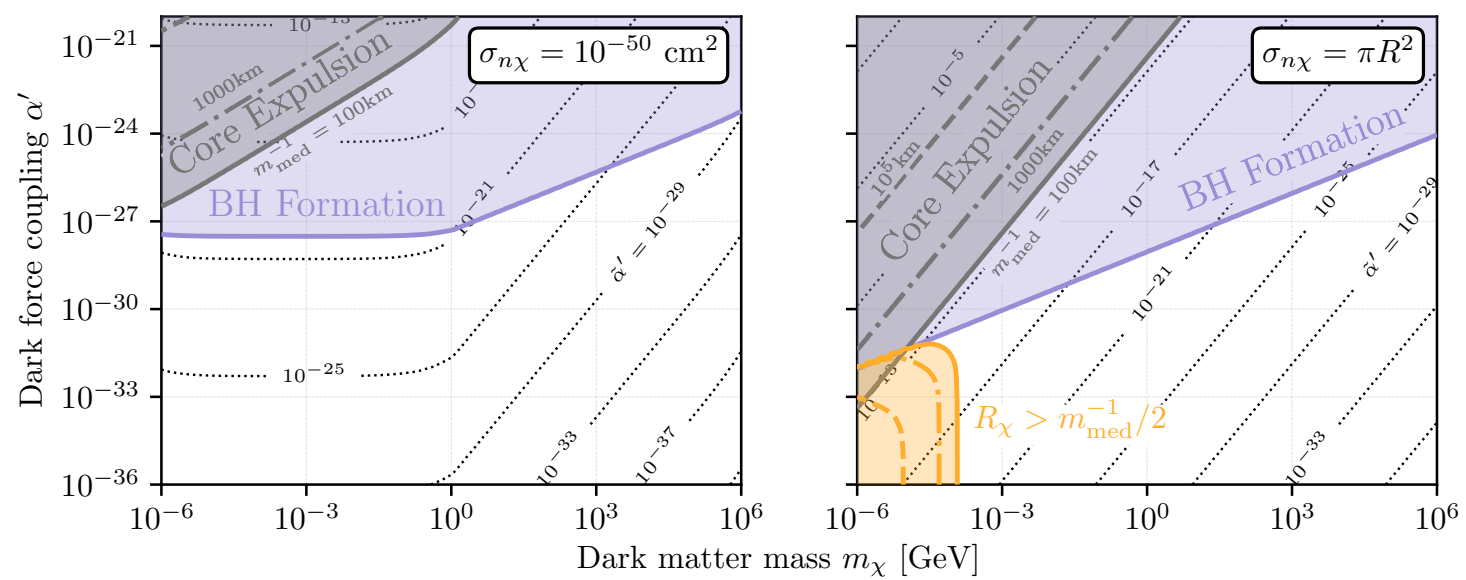

Figure 6. Constraints on an attractive dark force from a binary neutron star system as a function of the DM mass $m_{\chi}$ and the dark fine structure constant $\alpha^{\prime}$. The dotted contours show the value of $\widetilde{\alpha}^{\prime}$ (in the case of neutron stars with equal dark charge-over-mass ratio) or $\widetilde{\beta}^{\prime}$ (for the case that only one neutron star carries a dark charge, see eq. (2.2)) at each point in the plot. In the purple region, the dark force induces collapse of the neutron stars' dark cores to black holes, assuming the amount of DM inside the stars is determined by DM capture from the halo, see appendix A. Inside the gray shaded region, the dark cores are expelled from the neutron stars as soon as the screening of the force is lifted. We show this region for $m_{\text {med }}^{-1}=100 \mathrm{~km}$ (solid), $1000 \mathrm{~km}$ (dot-dashed), and $1 \times 10^{5} \mathrm{~km}$. Within the yellow regions, where $R_{\chi} \geq m_{\text {med }}^{-1} / 2$, the DM particles would be stripped away from their host neutron star before they can have a significant impact on the inspiral. The benchmark values of $m_{\text {med }}^{-1}$ are the same as for the DM expulsion bound. The neutron star parameters are chosen in accordance with table 1 , and without loss of generality we have chosen $q_{\chi}=1$. We have assumed a DM-nucleon scattering cross section $\sigma_{n \chi}=8 \times 10^{-47} \mathrm{~cm}^{2}$, a DM density in the vicinity of the neutron star of $\rho_{\chi}=0.3 \mathrm{GeV} / \mathrm{cm}^{3}$, and a DM velocity relative to the neutron star of $\bar{v}=220 \mathrm{~km} \mathrm{~s}^{-1}$.

For the parameter ranges that we are most interested in, namely those featuring the largest possible $N_{\chi}$ and $\alpha^{\prime}$, minimum stable $R_{\chi}$ values typically occur at relativistic $p_{F}$. In this case, eq. (4.1) can be simplified to the constraint

$$
N_{\chi}<\frac{3 \sqrt{\pi}}{2\left(\alpha^{\prime}+G_{N} m_{\chi}^{2}\right)^{3 / 2}} .
$$

This limit is saturated if the baryonic gravitational potential is completely negligible. If we assume that the maximum value of $N_{\chi}$ is determined by DM capture from the halo (see eq. (3.12)), we can turn eq. (4.2) into a constraint on $\widetilde{\alpha}^{\prime}$. Using the definition of $\widetilde{\alpha}^{\prime}$ from eq. (2.2), we obtain

$$
\widetilde{\alpha}^{\prime} \lesssim\left(\frac{9 \pi^{2}}{4}\right)^{1 / 3} \frac{1}{G_{N} M^{2}}\left(\frac{\pi R^{2} v_{\mathrm{esc}}^{2} t_{\mathrm{NS}} \rho_{\chi}}{\bar{v} m_{\chi}}\right)^{4 / 3}=6.7 \times 10^{-20}\left(\frac{1 \mathrm{GeV}}{m_{\chi}}\right)^{4 / 3} .
$$

Here, we have used the neutron star parameters from table 1 for both neutron stars.

The parameter values where no stable dark cores can exist, i.e. where eq. (4.1) has no solution, are shown in purple in figures 5 and 6 . In figure 5 , no assumptions are made on 
how the dark particles came to be within the neutron star. In figure 6 , the same constraints are shown as a function of $m_{\chi}$ and $\alpha^{\prime}$, assuming that the dark core has been accumulated via capture. The two panels correspond to different assumptions on the capture cross section $\sigma_{n \chi}$. We observe from figure 6 that at $m_{\chi} \gtrsim 1 \mathrm{GeV}$, larger $m_{\chi}$ implies more stringent constraints on $\alpha^{\prime}$. This is because of the lower DM number density at large $m_{\chi}$, which implies smaller total dark charge $Q_{\chi}$ and thus a smaller attractive force. Of course, Fermi repulsion is also weaker when less DM is accreted, however the corresponding contribution to the virial equation (4.1) scales less strongly with $m_{\chi}$ than the dark force potential. At

$m_{\chi} \lesssim 1 \mathrm{GeV}$, the purple exclusion curve and the $\widetilde{\alpha}^{\prime}$ contours in the left panel of figure 6 flatten because of Pauli blocking, which limits the number of final states available to the neutrons participating in scattering processes and thus limits the number of target particles that DM particles can scatter off and be captured.

The bounds from black hole formation shown in figures 5 and 6 have been derived assuming the dark core is a degenerate Fermi gas, i.e. that Fermi repulsion is maximal. It is possible that the dark core collapses to form a black hole before the required number of particles are captured to satisfy the degeneracy condition. This would lead to a more stringent bound in comparison to figures 5 and 6 . We have also neglected the question of whether the black hole formed would indeed consume the entire neutron star in finite time or if it evaporates. If the black hole evaporates before consuming the star, no residual dark core would be left, so in this case no modifications to the inspiral dynamics are expected. However, using the results of ref. [98], we conclude that evaporation is faster than the collapse of the neutron star only if $M_{\chi} \lesssim 1 \times 10^{-20} \mathrm{M}_{\odot}$.

Solving eq. (4.1) also yields the radius of the dark core when such a stable radius exists. For light DM mass, Fermi repulsion results in core radii $R_{\chi}$ larger than the neutron star radius $R$. For very light DM, $R_{\chi}$ becomes even larger than $m_{\text {med }}^{-1}$, so that the dynamics of the dark core and the binary inspiral would be very different to that of section 2 . The parameter region where this happens is shaded in yellow in figures 5 and 6 . As previously discussed in section 3.2, these yellow curves correspond to the maximum amount of charge that fits within the domain of influence of the neutron star. Particles at radii larger than $m_{\text {med }}^{-1}$ will be efficiently stripped off the neutron star.

\subsection{Expulsion of the dark core}

We can estimate whether the dark cores of two inspiraling neutron stars remain trapped at the center of their host stars, or whether they are ejected from them by virtue of the strong dark sector force acting on them. If the dark cores are ejected as soon as the dark force between the two neutron stars becomes unscreened the observed signal will be unchanged compared to the predictions of pure general relativity.

Consider the case where the dark core of one of the neutron stars is about to be expelled from the star and is located at its boundary. We compare the gravitational force experienced by the DM particles (which is strongest at the boundary of the neutron star) to the opposing dark force induced by the dark core of the other neutron star in the binary 
system:

$$
\begin{aligned}
\left|F_{\text {grav }, i}\right| & =\frac{G_{N} M_{i} M_{\chi, i}}{R_{i}^{2}}, \\
\left|F_{\text {dark }, i}\right| & =\frac{\alpha^{\prime} Q_{i} Q_{j}}{\Delta^{2}}\left(1+m_{\text {med }} \Delta\right) e^{-m_{\text {med }} \Delta} .
\end{aligned}
$$

Here the indices $i$ and $j$ label the two neutron stars $(i=1, j=2$ or $i=2, j=1)$. As before, $R_{i}$ are the neutron star radii, $\Delta$ is the distance between them, $M_{b, i}$ and $M_{\chi, i}$ are the total masses of their baryonic and dark constituents, respectively, $M_{i}=M_{b, i}+M_{\chi, i}$ are their total masses, and $Q_{i}$ are their dark charges. The dark core is expelled from the neutron star if $\left|F_{\text {grav }, i}\right|<\left|F_{\text {dark }, i}\right|$, i.e. if

$$
\left(\frac{\Delta}{R_{i}}\right)^{2}<\frac{\alpha^{\prime} Q_{i} Q_{j}\left(1+m_{\mathrm{med}} \Delta\right) e^{-m_{\mathrm{med}} \Delta}}{G_{N} M_{i} M_{\chi, i}} .
$$

In the regime $m_{\text {med }} \Delta \ll 1$, and for DM particles carrying unit charge under the dark force $\left(q_{\chi}=1\right)$, this condition simplifies to

$$
\left(\frac{\Delta}{R_{i}}\right)^{2}<\frac{\alpha^{\prime} N_{\chi, j}}{G_{N} M_{i} m_{\chi}} \simeq 10^{25} \sqrt{\alpha^{\prime} \widetilde{\alpha}^{\prime}}\left(\frac{1 \mathrm{keV}}{m_{\chi}}\right) .
$$

In the last equality, we have assumed that both neutron stars carry equal amounts of DM, and we have used the definition of $\widetilde{\alpha}^{\prime}$ from eq. (2.2). This implies that the gravitational potential can be overcome already at very large distances, leading to expulsion of the dark cores long before the actual merger.

In figures 5 and 6 we show the bounds arising from requiring that the expulsion distance be less than the force range as gray contours. These contours are calculated without neglecting Yukawa screening effects. We consider three different values for the range of the dark force, $m_{\text {med }}^{-1}$ at $m_{\text {med }}^{-1}=100 \mathrm{~km}$, the onset of the dark force would happen within the LIGO/VIRGO frequency band; at $m_{\text {med }}^{-1}=1000 \mathrm{~km}$, the dark force would be suppressed while the binary system emits gravitational waves in the lower frequency band, but unsuppressed when it emits in the LIGO/VIRGO band; at $m_{\text {med }}^{-1}=1 \times 10^{5} \mathrm{~km}$, the dark force would be important for both low frequency detectors and LIGO/VIRGO.

\subsection{Constraints on dark core production mechanisms}

As in the case of a repulsive dark force (section 3), further constraints exist on specific dark core production mechanisms. In figure 5, we indicate by the horizontal black dashed lines the maximum amount of dark matter that can be accreted from the halo assuming a geometric capture cross section (see section 3.3 and appendix A). Figure 6 also assumes DM particle capture, with either a fixed DM-nucleon scattering cross section (left panel) or a geometric capture cross section (right panel). The greatest possible value of $\widetilde{\alpha}^{\prime}$ that can be realized assuming the dark core is produced via accretion from the halo (eq. (3.12)) is $\widetilde{\alpha}^{\prime} \sim 10^{-14}$. It occurs for $m_{\chi}=0.1 \mathrm{MeV}$. These values are determined by calculating the $m_{\chi}$ value where the flat yellow region of figure 5 hits the accretion line and then plugging this value into the black hole formation bound. 
Producing dark sector particles in neutron decay is not a viable option for attractive dark forces. In the absence of such a dark force the neutron star equation of state is already too soft to allow for $2 M_{\odot}$ neutron stars [89, 90], and an attractive force will only further soften it.

The possibility that the dark core is produced radiatively during the supernova explosion that creates the neutron star is constrained in the same way as for repulsive dark forces, see section 3.4. This constraint is shown in figure 5 as a solid horizontal black line. The greatest possible value of $\widetilde{\alpha}^{\prime}$ for radiatively produced DM is 0.23 . It occurs for $m_{\chi}=0.25 \mathrm{GeV}$ under the assumption of perfectly efficient production in the supernova. Note again that these masses are well above the supernova temperature, thus it is implausible to expect a large fraction of the supernova energy to be converted to dark particles in order to achieve this upper-limit value.

In summary, we conclude from figure 5 that dark sector forces cannot significantly affect gravitational wave observations of neutron star inspirals as long as we assume that the neutron stars acquire their dark charge during or after their creation in a supernova. In principle, this no-go theorem could be avoided in tiny regions of parameter space (upper left corner of the plots in figure 5) by a mechanism that endows neutron stars with dark cores that account for at least $\sim 1 \%$ of their mass. Such a massive DM core could be realized if the DM is already collapsed into a very dense structure, and subsequently the entire DM core either seeds the formation of a star or is captured en masse by a star or neutron star in what must be an incredibly rare event. Of course, such a mechanism is unavailable to repulsively-interacting DM, as the dark sector interactions would prevent such collapse.

Both of these scenarios are constrained by the requirements that the collapse of pure DM systems not damage the observed overall halo structure of galaxies. In particular, collapsing and asymmetrically charged DM objects should only account for a small fraction of the DM in the Universe, making the envisioned capture events even more rare. A detailed analysis of the abundance bounds in this case is left to a future publication. Assuming nonetheless that the neutron star consists of equal parts DM and baryons, i.e. $M_{\chi} \sim M_{b}$, the maximum value of $\widetilde{\alpha}^{\prime} \sim 0.3$ occurs for $m_{\chi} \sim 1 \mathrm{GeV}$ and $\alpha^{\prime} \sim G_{N} m_{\chi}^{2}$, with the amount of DM particles just below that which would cause collapse to a black hole.

Since compact dark objects that have collapsed in the early Universe are scarce, it is likely in the above scenarios that a neutron stars carrying a large dark charge forms a binary system with an uncharged partner. Therefore, in these scenarios, it is likely that the dominant signature of the dark force would be due to dipole radiation (see section 2.2).

\section{Conclusions}

We have investigated the conditions under which a new long-range force acting on dark matter can affect the dynamics of neutron star inspirals and can lead to observable modifications to gravitational wave signals observed in gravitational wave detectors such as LIGO/VIRGO. This scenario is based on the assumption that a large population of DM particles exists within neutron stars. 
For repulsive forces, a crucial constraint arises from the requirement that the dark core of the neutron star remains stable (i.e. that gravitational attraction remains stronger than the repulsive dark force). Moreover, such scenarios are severely limited by the fact that a sufficiently large dark core cannot be produced via DM accretion from the halo; the greatest effect on inspirals which is possible through accretion has strength normalized to gravity of $\widetilde{\alpha}^{\prime}=2 \times 10^{-15}$. Considering more exotic production mechanisms for the DM particles, we conclude that the strongest possible signal in the case of a repulsive dark force corresponds to an $\widetilde{\alpha}^{\prime}$ value of 0.071 , and arises through neutron decays similar to those which might explain the neutron lifetime measurement disagreements.

For attractive dark forces additional constraints arise from the possible collapse of a massive dark core to a black hole that would consume the whole neutron star. In addition, a strong attractive force could lead to the expulsion of the dark cores from their host neutron stars in a binary system. In this case, the dark cores and their self-interaction cannot contribute significantly to gravitational wave signals any more. A similar effect is the stripping of DM particles from the neutron stars in a binary system. In fact, if the DM population bound to a neutron star consists of relatively light particles, it may extend to radii much larger than that of the baryonic matter. Such DM particles are easily freed from the star's binding force and thus stop contributing to the inspiral dynamics.

In the case of attractive dark forces, the maximum effect on the inspiral dynamics and the gravitational wave signals also depends on the mechanisms by which the dark cores of the neutron stars form. For accretion from the halo, the maximum possible effect is characterized by $\widetilde{\alpha}^{\prime}=10^{-14}$. If the DM population is produced in the supernova that also gives birth to the neutron star, the strongest possible signal is given by $\widetilde{\alpha}^{\prime}=0.04$. Even stronger modifications to gravitational wave signals are possible up to $\widetilde{\alpha}^{\prime}=0.3$. This value, however, can only be realized if the DM particles have either seeded the formation of the neutron star's progenitor star or have been accreted as an already collapsed core in a very rare process.

We conclude that, if any deviations from the predictions of general relativity are found by LIGO/ VIRGO in gravitational wave signals from neutron star inspirals, new exotic mechanisms of DM production in neutron stars are required. This would likely herald the existence of large, compact DM structures that either seed star formation or are captured by stars or neutron stars later in their lives. It has been shown in ref. [70] that future gravitational wave telescopes like the Einstein Telescope can substantially improve the new physics sensitivity following a similar search strategy.

We finally note that, beyond the observables discussed in this paper, the dynamics of the dark sector could also affect gravitational waves from binary neutron star inspirals in very different ways. Namely, rather than altering the force between the two inspiraling bodies, DM particles could instead alter the properties of the neutron stars. This includes, for instance, alterations in their tidal deformability due to the presence of dark sector particles [22] or alterations arising from particles produced by black hole superradiance effects [99]. Neither of these effects would contradict the results presented here for probing dark sector forces. 


\section{Acknowledgments}

The authors gratefully acknowledge helpful discussions with Chen Sun and Jun Zhang. We especially want to thank Ian Harry for many useful comments on the manuscript. The work of WS was supported by the Alexander von Humboldt Foundation in the framework of the Sofja Kovalevskaja Award 2016, endowed by the German Federal Ministry of Education and Research. JK, RL, and TO have received funding from the German Research Foundation (DFG) under Grant Nos. EXC-1098, FOR 2239 and GRK 1581, and from the European Research Council (ERC) under the European Union's Horizon 2020 research and innovation programme (grant agreement No. 637506, " $\nu$ Directions"). JK and TO would like to thank the CERN Theoretical Physics Department for hospitality and support.

\section{A Capture rate calculation}

The capture rates for dark particles in the scenarios under consideration in this paper have been calculated in refs. [35, 45, 100, 101]. Here, we summarize these calculations, fixing the notation and highlighting the various assumptions made. We emphasize that, while we have utilized standard DM halo parameters throughout this article as a benchmark, our results do not require that the particles captured in neutron stars make up all the DM in the Universe.

Firstly, we approximate that the average DM velocity, escape velocity, and baryon density are uniform throughout the body of the neutron star and that the DM-nucleon scattering cross section is velocity-independent. Then, the capture rate of DM on a neutron star is

$$
C_{\chi} \simeq \sqrt{\frac{6}{\pi}} \frac{\rho_{\chi}}{m_{\chi}} \frac{1}{\bar{v}} \frac{v(r)^{2}}{1-v(r)^{2}} f\left(\sigma_{n \chi}\right) \xi N_{B}\left[1-\frac{1-e^{-B^{2}}}{B^{2}}\right] .
$$

The parameters and functions that enter this equation and have not already been defined are:

- $v(r)$ is the infall speed of the dark particles, which we approximate by the escape velocity at the surface of the neutron star. For compact objects like neutron stars, this is a good approximation because the kinetic energy a dark particle acquires during infall is much larger than the initial kinetic energy at $r=\infty$.

- The factor $1-v(r)^{2}$ in the denominator arises from general relativity corrections in the vicinity of the neutron star [30]. These leads to a $\sim 60 \%$ increase of the capture rate for the neutron star parameters in table 1.

- $\xi$ is the fraction of neutrons that contribute in the scatting after including the effects of Pauli blocking. $\xi=1$ for DM masses above a $\mathrm{GeV}$, but for smaller values where the momentum of the DM particle becomes smaller than the neutron Fermi momentum $p_{F}=\left(3 \pi^{2} \rho_{n} / m_{n}\right)^{1 / 3}$ (given here in terms of the neutron mass density $\rho_{n}$ and the neutron mass $m_{n}$ ) the fraction of neutrons which have enough momentum to have an accessible final state to scatter in to is

$$
\xi \simeq \frac{\sqrt{2} m_{r} v_{\mathrm{esc}}}{p_{F}} .
$$


Here $m_{r}$ is the reduced mass of the neutron-DM system, $m_{r}=m_{\chi} m_{n} /\left(m_{\chi}+m_{n}\right)$.

- $f\left(\sigma_{n \chi}\right)$ is a function of the DM-nucleon cross section that determines the probability of scattering:

$$
f\left(\sigma_{n \chi}\right)=\sigma_{\text {sat }}\left(1-e^{-\sigma_{n \chi} / \sigma_{\text {sat }}}\right) .
$$

For the saturation cross-section we use the result of ref. [30]

$$
\sigma_{\mathrm{sat}} \simeq \frac{R^{2}}{0.45 N_{b} \xi},
$$

where $N_{b}$ is the number of baryons in the neutron star.

- $B$ is a function accounting for the minimum energy loss necessary to capture a DM particle:

$$
B=\frac{6 v_{\mathrm{esc}}^{2}}{\bar{v}^{2}} \frac{m_{\chi} m_{n}}{\left(m_{\chi}-m_{n}\right)^{2}} .
$$

This function is smaller than one only when the DM mass is $\gtrsim 10^{6} \mathrm{GeV}$.

Using $C_{\chi}$ from eq. (A.1), the number of DM particles captured will be

$$
N_{\chi}=C_{\chi} t_{\mathrm{NS}}
$$

Note that we here neglect the possibility of DM self-annihilation, co-annihilation [45] or semi-annihilation [102], which would reduce $N_{\chi}$.

Open Access. This article is distributed under the terms of the Creative Commons Attribution License (CC-BY 4.0), which permits any use, distribution and reproduction in any medium, provided the original author(s) and source are credited.

\section{References}

[1] Virgo and Ligo Scientific collaborations, B.P. Abbott et al., Observation of gravitational waves from a binary black hole merger, Phys. Rev. Lett. 116 (2016) 061102 [arXiv: 1602.03837] [INSPIRE].

[2] Virgo and Ligo Scientific collaborations, B.P. Abbott et al., Binary black hole mergers in the first advanced LIGO observing run, Phys. Rev. X 6 (2016) 041015 [arXiv: 1606.04856] [INSPIRE].

[3] Virgo and LigO Scientific collaborations, B.P. Abbott et al., GW151226: observation of gravitational waves from a 22-solar-mass binary black hole coalescence, Phys. Rev. Lett. 116 (2016) 241103 [arXiv: 1606.04855] [INSPIRE].

[4] Virgo and LigO Scientific collaborations, B. Abbott et al., GW170817: observation of gravitational waves from a binary neutron star inspiral, Phys. Rev. Lett. 119 (2017) 161101 [arXiv: 1710.05832] [INSPIRE].

[5] D.A. Coulter et al., Swope supernova survey 2017a (SSS17a), the optical counterpart to a gravitational wave source, Science 358 (2017) 1556 [arXiv:1710.05452] [INSPIRE]. 
[6] Virgo, Fermi-GBM, INTEGRAL and Ligo Scientific collaborations, B.P. Abbott et al., Gravitational waves and gamma-rays from a binary neutron star merger: GW170817 and GRB 170817A, Astrophys. J. 848 (2017) L13 [arXiv:1710.05834] [INSPIRE].

[7] Grond, SAlt Group, OzGrav, DFN, Integral, Virgo, Insight-Hxmt, MAXi TeAm, Fermi-LAT, J-GEM, RATIR, ICECube, CAASTRO, LWA, ePESSTO, GRAWITA, RIMAS, SKA South Africa/MeerKAT, H.E.S.S., 1M2H Team, IKI-GW Follow-up, Fermi GBM, Pi of Sky, DWF (Deeper Wider Faster Program), Dark Energy Survey, Master, Astrosat Cadmium Zinc Telluride Imager Team, Swift, Pierre Auger, ASKap, Vinrouge, JAGWAR, Chandra Team at McGill University, TTU-NRAO, GROWTH, AgIle Team, MWA, ATCA, ASt3, TOROS, Pan-STARrs, NuStar, ATlas Telescopes, BoOtes, CaltechnraO, Ligo Scientific, High Time Resolution Universe Survey, Nordic Optical Telescope, las Cumbres Observatory Group, tZaC Consortium, LOFAR, IPN, Dlt40, Texas Tech University, HAWC, ANTARES, Ku, Dark Energy Camera GW-EM, CAlet, Euro Vlbi Team and Alma collaborations, B.P. Abbott et al., Multi-messenger observations of a binary neutron star merger, Astrophys. J. 848 (2017) L12 [arXiv:1710.05833] [INSPIRE].

[8] I.H. Stairs, Testing general relativity with pulsar timing, Living Rev. Rel. 6 (2003) 5 [astro-ph/0307536] [INSPIRE].

[9] A. Belenchia, M. Letizia, S. Liberati and E.D. Casola, Higher-order theories of gravity: diagnosis, extraction and reformulation via non-metric extra degrees of freedom - a review, Rept. Prog. Phys. 81 (2018) 036001 [arXiv: 1612.07749] [INSPIRE].

[10] R.-G. Cai, Z. Cao, Z.-K. Guo, S.-J. Wang and T. Yang, The gravitational-wave physics, Natl. Sci. Rev. 4 (2017) 687 [arXiv:1703.00187] [INSPIRE].

[11] L. Sagunski et al., Neutron star mergers as a probe of modifications of general relativity with finite-range scalar forces, Phys. Rev. D 97 (2018) 064016 [arXiv: 1709.06634] [INSPIRE].

[12] C. Burrage and J. Sakstein, Tests of chameleon gravity, Living Rev. Rel. 21 (2018) 1 [arXiv: 1709.09071] [INSPIRE].

[13] S. Nojiri and S.D. Odintsov, Cosmological bound from the neutron star merger GW170817 in scalar-tensor and $F(R)$ gravity theories, Phys. Lett. B 779 (2018) 425 [arXiv: 1711.00492] [INSPIRE].

[14] A. Dima and F. Vernizzi, Vainshtein screening in scalar-tensor theories before and after GW170817: constraints on theories beyond Horndeski, Phys. Rev. D 97 (2018) 101302 [arXiv: 1712.04731] [INSPIRE].

[15] E. Berti, K. Yagi and N. Yunes, Extreme gravity tests with gravitational waves from compact binary coalescences: (I) inspiral-merger, Gen. Rel. Grav. 50 (2018) 46 [arXiv: 1801.03208] [INSPIRE].

[16] Y. Gong, S. Hou, D. Liang and E. Papantonopoulos, Gravitational waves in Einstein-aether and generalized TeVeS theory after GW170817, Phys. Rev. D 97 (2018) 084040 [arXiv: 1801.03382] [INSPIRE].

[17] E. Berti, K. Yagi, H. Yang and N. Yunes, Extreme gravity tests with gravitational waves from compact binary coalescences: (II) ringdown, Gen. Rel. Grav. 50 (2018) 49 [arXiv: 1801.03587] [INSPIRE]. 
[18] J. Abedi and N. Afshordi, Echoes from the abyss: a highly spinning black hole remnant for the binary neutron star merger GW170817, arXiv:1803.10454 [INSPIRE].

[19] A. Hook and J. Huang, Probing axions with neutron star inspirals and other stellar processes, JHEP 06 (2018) 036 [arXiv:1708.08464] [INSPIRE].

[20] D. Croon, A.E. Nelson, C. Sun, D.G.E. Walker and Z.-Z. Xianyu, Hidden-sector spectroscopy with gravitational waves from binary neutron stars, Astrophys. J. 858 (2018) L2 [arXiv: 1711.02096] [INSPIRE].

[21] J. Ellis et al., Search for dark matter effects on gravitational signals from neutron star mergers, Phys. Lett. B 781 (2018) 607 [arXiv:1710.05540] [INSPIRE].

[22] A. Nelson, S. Reddy and D. Zhou, Dark halos around neutron stars and gravitational waves, arXiv: 1803.03266 [INSPIRE].

[23] J. Ellis, G. Hütsi, K. Kannike, L. Marzola, M. Raidal and V. Vaskonen, Dark matter effects on neutron star properties, Phys. Rev. D 97 (2018) 123007 [arXiv:1804.01418] [InSPIRE].

[24] E.G. Adelberger, J.H. Gundlach, B.R. Heckel, S. Hoedl and S. Schlamminger, Torsion balance experiments: a low-energy frontier of particle physics, Prog. Part. Nucl. Phys. 62 (2009) 102 [INSPIRE].

[25] E.J. Salumbides, W. Ubachs and V.I. Korobov, Bounds on fifth forces at the sub-Angstrom length scale, J. Molec. Spectrosc. 300 (2014) 65 [arXiv: 1308.1711] [INSPIRE].

[26] E.J. Salumbides, J.C.J. Koelemeij, J. Komasa, K. Pachucki, K.S.E. Eikema and W. Ubachs, Bounds on fifth forces from precision measurements on molecules, Phys. Rev. D 87 (2013) 112008 [arXiv: 1304.6560] [INSPIRE].

[27] J. Bergé, P. Brax, G. Métris, M. Pernot-Borràs, P. Touboul and J.-P. Uzan, MICROSCOPE mission: first constraints on the violation of the weak equivalence principle by a light scalar dilaton, Phys. Rev. Lett. 120 (2018) 141101 [arXiv:1712.00483] [INSPIRE].

[28] P. Fayet, MICROSCOPE limits for new long-range forces and implications for unified theories, Phys. Rev. D 97 (2018) 055039 [arXiv:1712.00856] [InSPIRE].

[29] I. Goldman and S. Nussinov, Weakly interacting massive particles and neutron stars, Phys. Rev. D 40 (1989) 3221 [INSPIRE].

[30] C. Kouvaris, WIMP annihilation and cooling of neutron stars, Phys. Rev. D 77 (2008) 023006 [arXiv: 0708.2362] [INSPIRE].

[31] F. Sandin and P. Ciarcelluti, Effects of mirror dark matter on neutron stars, Astropart. Phys. 32 (2009) 278 [arXiv:0809.2942] [INSPIRE].

[32] C. Kouvaris and P. Tinyakov, Can neutron stars constrain dark matter?, Phys. Rev. D 82 (2010) 063531 [arXiv: 1004.0586] [INSPIRE].

[33] A. de Lavallaz and M. Fairbairn, Neutron stars as dark matter probes, Phys. Rev. D 81 (2010) 123521 [arXiv:1004.0629] [INSPIRE].

[34] P. Ciarcelluti and F. Sandin, Have neutron stars a dark matter core?, Phys. Lett. B 695 (2011) 19 [arXiv: 1005. 0857] [INSPIRE].

[35] S.D. McDermott, H.-B. Yu and K.M. Zurek, Constraints on scalar asymmetric dark matter from black hole formation in neutron stars, Phys. Rev. D 85 (2012) 023519 [arXiv:1103.5472] [INSPIRE]. 
[36] C. Kouvaris and P. Tinyakov, Excluding light asymmetric bosonic dark matter, Phys. Rev. Lett. 107 (2011) 091301 [arXiv: 1104.0382] [INSPIRE].

[37] S.C. Leung, M.C. Chu and L.M. Lin, Dark-matter admixed neutron stars, Phys. Rev. D 84 (2011) 107301 [arXiv:1111.1787] [INSPIRE].

[38] C. Kouvaris, Limits on self-interacting dark matter, Phys. Rev. Lett. 108 (2012) 191301 [arXiv:1111.4364] [INSPIRE].

[39] T. Güver, A.E. Erkoca, M. Hall Reno and I. Sarcevic, On the capture of dark matter by neutron stars, JCAP 05 (2014) 013 [arXiv:1201.2400] [INSPIRE].

[40] X. Li, F. Wang and K.S. Cheng, Gravitational effects of condensate dark matter on compact stellar objects, JCAP 10 (2012) 031 [arXiv:1210.1748] [INSPIRE].

[41] J. Bramante, K. Fukushima and J. Kumar, Constraints on bosonic dark matter from observation of old neutron stars, Phys. Rev. D 87 (2013) 055012 [arXiv:1301.0036] [INSPIRE].

[42] N.F. Bell, A. Melatos and K. Petraki, Realistic neutron star constraints on bosonic asymmetric dark matter, Phys. Rev. D 87 (2013) 123507 [arXiv:1301.6811] [INSPIRE].

[43] S.C. Leung, M.C. Chu, L.M. Lin and K.W. Wong, Dark-matter admixed white dwarfs, Phys. Rev. D 87 (2013) 123506 [arXiv:1305.6142] [INSPIRE].

[44] I. Goldman, R.N. Mohapatra, S. Nussinov, D. Rosenbaum and V. Teplitz, Possible implications of asymmetric fermionic dark matter for neutron stars, Phys. Lett. B 725 (2013) 200 [arXiv: 1305.6908] [INSPIRE].

[45] J. Bramante, K. Fukushima, J. Kumar and E. Stopnitzky, Bounds on self-interacting fermion dark matter from observations of old neutron stars, Phys. Rev. D 89 (2014) 015010 [arXiv:1310.3509] [INSPIRE].

[46] H. Zheng, K.-J. Sun and L.-W. Chen, Old neutron stars as probes of isospin-violating dark matter, Astrophys. J. 800 (2015) 141 [arXiv: 1408.2926] [INSPIRE].

[47] S. Mukhopadhyay, D. Atta, K. Imam, D.N. Basu and C. Samanta, Compact bifluid hybrid stars: hadronic matter mixed with self-interacting fermionic asymmetric dark matter, Eur. Phys. J. C 77 (2017) 440 [Erratum ibid. C 77 (2017) 553] [arXiv: 1612.07093] [INSPIRE].

[48] M. Baryakhtar, J. Bramante, S.W. Li, T. Linden and N. Raj, Dark kinetic heating of neutron stars and an infrared window on WIMPs, SIMPs and pure higgsinos, Phys. Rev. Lett. 119 (2017) 131801 [arXiv: 1704.01577] [INSPIRE].

[49] N. Raj, P. Tanedo and H.-B. Yu, Neutron stars at the dark matter direct detection frontier, Phys. Rev. D 97 (2018) 043006 [arXiv: 1707.09442] [INSPIRE].

[50] M. Cermeño, M.A. Pérez-García and J. Silk, Fermionic light dark matter particles and the new physics of neutron stars, Publ. Astron. Soc. Austral. 34 (2017) e043 [arXiv: 1710.06866] [INSPIRE].

[51] Z. Rezaei, Neutron stars with spin polarized self-interacting dark matter, Astropart. Phys. 101 (2018) 1 [arXiv: 1803.08075] [INSPIRE].

[52] C. Kouvaris, P. Tinyakov and M.H.G. Tytgat, Non-primordial solar mass black holes, arXiv: 1804.06740 [INSPIRE]. 
[53] X.D. Wang, B. Qi, N.B. Zhang and S.Y. Wang, The maximum mass of dark matter existing in compact stars based on the self-interacting fermionic model, arXiv:1805.01314 [INSPIRE].

[54] C.-S. Chen and Y.-H. Lin, Reheating neutron stars with the annihilation of self-interacting dark matter, JHEP 08 (2018) 069 [arXiv: 1804.03409] [INSPIRE].

[55] D. Krause, H.T. Kloor and E. Fischbach, Multipole radiation from massive fields: application to binary pulsar systems, Phys. Rev. D 49 (1994) 6892 [INSPIRE].

[56] S. Mohanty and P. Kumar Panda, Particle physics bounds from the Hulse-Taylor binary, Phys. Rev. D 53 (1996) 5723 [hep-ph/9403205] [INSPIRE].

[57] M. Kaplinghat, S. Tulin and H.-B. Yu, Dark matter halos as particle colliders: unified solution to small-scale structure puzzles from dwarfs to clusters, Phys. Rev. Lett. 116 (2016) 041302 [arXiv: 1508.03339] [INSPIRE].

[58] S. Tulin and H.-B. Yu, Dark matter self-interactions and small scale structure, Phys. Rept. 730 (2018) 1 [arXiv: 1705. 02358] [inSPIRE].

[59] E. Braaten, D. Kang and R. Laha, Production of dark-matter bound states in the early universe by three-body recombination, arXiv:1806.00609 [INSPIRE].

[60] M. Pitkin, S. Reid, S. Rowan and J. Hough, Gravitational wave detection by interferometry (ground and space), Living Rev. Rel. 14 (2011) 5 [arXiv:1102.3355] [INSPIRE].

[61] C.M. Will, The confrontation between general relativity and experiment, Living Rev. Rel. 17 (2014) 4 [arXiv:1403.7377] [INSPIRE].

[62] K. Petraki and R.R. Volkas, Review of asymmetric dark matter, Int. J. Mod. Phys. A 28 (2013) 1330028 [arXiv: 1305.4939] [INSPIRE].

[63] K.M. Zurek, Asymmetric dark matter: theories, signatures and constraints, Phys. Rept. 537 (2014) 91 [arXiv: 1308.0338] [INSPIRE].

[64] P.C. Peters, Gravitational radiation and the motion of two point masses, Phys. Rev. 136 (1964) B1224 [INSPIRE].

[65] Virgo and LIGO Scientific collaborations, B.P. Abbott et al., The basic physics of the binary black hole merger GW150914, Annalen Phys. 529 (2017) 1600209 [arXiv: 1608.01940] [INSPIRE].

[66] H. Mathur, K. Brown and A. Lowenstein, An analysis of the LIGO discovery based on introductory physics, Am. J. Phys. 85 (2017) 676 [arXiv:1609.09349] [InSPIRE].

[67] B.P. Abbott et al., Sensitivity of the advanced LIGO detectors at the beginning of gravitational wave astronomy, Phys. Rev. D 93 (2016) 112004 [Addendum ibid. D 97 (2018) 059901] [arXiv: 1604.00439] [INSPIRE].

[68] M. Maggiore, Gravitational waves. Volume 1: theory and experiments, Oxford University Press, Oxford, U.K., (2008).

[69] A. Ross, Multipole expansion at the level of the action, Phys. Rev. D 85 (2012) 125033 [arXiv: 1202.4750] [INSPIRE].

[70] S. Alexander, E. McDonough, R. Sims and N. Yunes, Hidden-sector modifications to gravitational waves from binary inspirals, arXiv:1808.05286 [INSPIRE].

[71] H. Goldstein, Classical mechanics, Addison-Wesley series in physics, Addison-Wesley, U.S.A., (1980). 
[72] W. Lucha and F.F. Schoberl, The relativistic virial theorem, Phys. Rev. Lett. 64 (1990) 2733 [INSPIRE].

[73] M. Rose, Relativistic electron theory, John Wiley \& Sons, New York, U.S.A., (1961).

[74] K. Griest, Effect of the sun's gravity on the distribution and detection of dark matter near the earth, Phys. Rev. D 37 (1988) 2703 [InSPIRE].

[75] Kamiokande-II collaboration, K. Hirata et al., Observation of a neutrino burst from the supernova SN 1987a, Phys. Rev. Lett. 58 (1987) 1490 [inSPIRE].

[76] K.S. Hirata et al., Observation in the kamiokande-II detector of the neutrino burst from supernova SN 1987a, Phys. Rev. D 38 (1988) 448 [inSPIRE].

[77] R.M. Bionta et al., Observation of a neutrino burst in coincidence with supernova SN 1987 a in the large magellanic cloud, Phys. Rev. Lett. 58 (1987) 1494 [INSPIRE].

[78] IMB collaboration, C.B. Bratton et al., Angular distribution of events from SN 1987 , Phys. Rev. D 37 (1988) 3361 [inSPIRE].

[79] E.N. Alekseev, L.N. Alekseeva, I.V. Krivosheina and V.I. Volchenko, Detection of the neutrino signal from SN 1987 a in the LMC using the Inr Baksan underground scintillation telescope, Phys. Lett. B 205 (1988) 209 [InSPIRE].

[80] G.G. Raffelt, Stars as laboratories for fundamental physics, Chicago Univ. Pr., Chicago, U.S.A., (1996) [INSPIRE].

[81] E. Rrapaj and S. Reddy, Nucleon-nucleon bremsstrahlung of dark gauge bosons and revised supernova constraints, Phys. Rev. C 94 (2016) 045805 [arXiv:1511.09136] [inSPIRE].

[82] J.H. Chang, R. Essig and S.D. McDermott, Revisiting supernova 1987 a constraints on dark photons, JHEP 01 (2017) 107 [arXiv:1611.03864] [INSPIRE].

[83] E. Hardy and R. Lasenby, Stellar cooling bounds on new light particles: plasma mixing effects, JHEP 02 (2017) 033 [arXiv:1611.05852] [INSPIRE].

[84] C. Mahoney, A.K. Leibovich and A.R. Zentner, Updated constraints on self-interacting dark matter from supernova 1987a, Phys. Rev. D 96 (2017) 043018 [arXiv:1706.08871] [INSPIRE].

[85] J.H. Chang, R. Essig and S.D. McDermott, Supernova 1987 a constraints on sub-GeV dark sectors, millicharged particles, the QCD axion and an axion-like particle, JHEP 09 (2018) 051 [arXiv: 1803.00993] [INSPIRE].

[86] R. Laha and J.F. Beacom, Gadolinium in water Cherenkov detectors improves detection of supernova $\nu_{e}$, Phys. Rev. D 89 (2014) 063007 [arXiv: 1311.6407] [INSPIRE].

[87] R. Laha, J.F. Beacom and S.K. Agarwalla, New power to measure supernova $\nu_{e}$ with large liquid scintillator detectors, arXiv:1412.8425 [INSPIRE].

[88] A. Nikrant, R. Laha and S. Horiuchi, Robust measurement of supernova $\nu_{e}$ spectra with future neutrino detectors, Phys. Rev. D 97 (2018) 023019 [arXiv:1711.00008] [INSPIRE].

[89] D. McKeen, A.E. Nelson, S. Reddy and D. Zhou, Neutron stars exclude light dark baryons, Phys. Rev. Lett. 121 (2018) 061802 [arXiv:1802.08244] [INSPIRE].

[90] J.M. Cline and J.M. Cornell, Dark decay of the neutron, JHEP 07 (2018) 081 [arXiv: 1803.04961] [INSPIRE]. 
[91] G. Baym, D.H. Beck, P. Geltenbort and J. Shelton, Testing dark decays of baryons in neutron stars, Phys. Rev. Lett. 121 (2018) 061801 [arXiv:1802.08282] [INSPIRE].

[92] T.F. Motta, P.A.M. Guichon and A.W. Thomas, Implications of neutron star properties for the existence of light dark matter, J. Phys. G 45 (2018) 05LT01 [arXiv:1802.08427] [INSPIRE].

[93] D. McKeen and A.E. Nelson, CP violating baryon oscillations, Phys. Rev. D 94 (2016) 076002 [arXiv: 1512.05359] [INSPIRE].

[94] B. Fornal and B. Grinstein, Dark matter interpretation of the neutron decay anomaly, Phys. Rev. Lett. 120 (2018) 191801 [arXiv:1801.01124] [INSPIRE].

[95] H. Ejiri and J.D. Vergados, Neutron disappearance inside the nucleus, arXiv:1805.04477 [INSPIRE].

[96] D.R. Lorimer and M. Kramer, Handbook of pulsar astronomy, Cambridge University Press, Cambridge, U.K., (2004).

[97] R.N. Manchester, G.B. Hobbs, A. Teoh and M. Hobbs, The Australia telescope national facility pulsar catalogue, Astron. J. 129 (2005) 1993 [astro-ph/0412641] [INSPIRE].

[98] F. Capela, M. Pshirkov and P. Tinyakov, Constraints on primordial black holes as dark matter candidates from capture by neutron stars, Phys. Rev. D 87 (2013) 123524 [arXiv: 1301.4984] [INSPIRE].

[99] D. Baumann, H.S. Chia and R.A. Porto, Probing ultralight bosons with binary black holes, arXiv: 1804.03208 [INSPIRE].

[100] A. Gould, WIMP distribution in and evaporation from the sun, Astrophys. J. 321 (1987) 560 [INSPIRE].

[101] A. Gould, Resonant enhancements in WIMP capture by the earth, Astrophys. J. 321 (1987) 571 [INSPIRE].

[102] F. D'Eramo and J. Thaler, Semi-annihilation of dark matter, JHEP 06 (2010) 109 [arXiv: 1003.5912] [INSPIRE]. 